\title{
A New Game Equivalence, its Logic and Algebra
}

\author{
Johan van Benthem ${ }^{1,2,3}$. Nick Bezhanishvili ${ }^{1}$ - Sebastian Enqvist ${ }^{2,4}$
}

Received: 20 February 2018 / Accepted: 2 October 2018 / Published online: 9 November 2018

(C) The Author(s) 2018

\begin{abstract}
We present a new notion of game equivalence that captures basic powers of interacting players. We provide a representation theorem, a complete logic, and a new game algebra for basic powers. In doing so, we establish connections with imperfect information games and epistemic logic. We also identify some new open problems concerning logic and games.
\end{abstract}

Keywords Games · Modal logic $\cdot$ Neighbourhood semantics · Basic power · Imperfect information

\section{Introduction}

Games are a basic model for interactive agency, but how much structure do we need? Game theory offers strategic form games and extensive games, representing different levels of detail. Logicians have studied further levels, such as powers of players over the outcomes of a game. Each of these levels comes with a notion of invariance between structures that matches a logical language - and as in many fields, the search

\footnotetext{
Nick Bezhanishvili

N.Bezhanishvili@uva.nl

Johan van Benthem

J.vanBenthem@uva.nl

Sebastian Enqvist

thesebastianenqvist@gmail.com
}

1 Institute for Logic, Language and Computation, University of Amsterdam, P.O. Box 94242, 1090 GE Amsterdam, The Netherlands

2 Stanford University, Stanford, CA, USA

3 Tsinghua University, Beijing, China

4 Stockholm University, Filosofiska Institutionen, 106 91, Stockholm, Sweden 
for new invariances is ongoing. In this paper we offer a new notion bridging between game theory and logic: basic power equivalence, that uses powers encoding a sort of qualitative equilibria where all players matter. We determine its properties in a new representation theorem, find a complete associated modal neighborhood logic of a novel kind, and explore a new game algebra for basic powers that eventually forces us to change from functional to relational strategies. Moreover, we establish interesting connections with imperfect information games and epistemic logic.

This paper fits with a body of earlier work. Our approach is partly inspired by the computational literature on process equivalences, ranging from coarser trace equivalence to more fine-grained notions of bisimulation [5]. Even more central to us is the notion of power equivalence, implicit in the game algebra of Parikh [19], which also links with the set-theoretic forms for games in [8]. A precursor inside game theory is the celebrated transformation analysis of equivalent games with imperfect information by Thompson [23], refined in [10]. Game theory also has comparative discussions of the information available in extensive forms and in strategic normal forms [17], a style of invariance analysis that remains to be connected to our logic-based approach.

Another highly relevant strand for what follows is the work on social rights and games starting from [12], developed technically by Peleg and co-authors in a sequence of papers and books, cf. [21, 22], and connected to modal coalition logics by Pauly in [20], with further contributions such as [14]. More specific references to this tradition will be found at various places in this paper.

Even so, our analysis has clear limitations in what it takes on board. More delicate intuitions of game equivalence emerge once we consider players' preferences, or their types, or when we focus on correlations between available equilibria in the games being compared. These richer settings are beyond the scope of this paper, but they pose a natural challenge to logic-based approaches.

\section{Equivalence of Games, Old and New Answers}

The question when two games are considered equivalent is fundamental to any game theory. And as in many areas of mathematics, there is no unique answer: different natural candidates exist. Here is an example from van Benthem [1].

Example 2.1 Are the following two games the same?
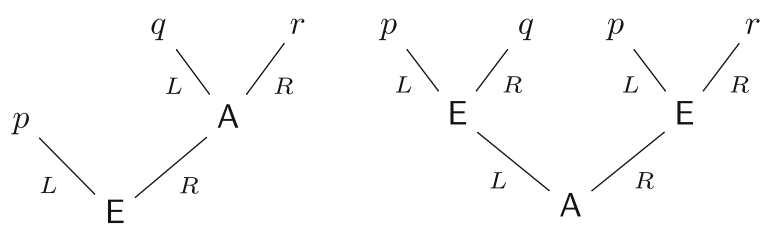

Fig. 1 Equivalent games 
There are two players A, E, whose turns are indicated at nodes in the extensive game trees, and the proposition letters $p, q, r$ mark the outcomes at the end of the game. Here are two different natural ways of answering the stated question.

Note In what follows, we assume familiarity with basic notions from game theory such as extensive game tree, strategy, outcome, games in strategic form, perfect information, and imperfect information. For cases where more precision matters in the text, we have collected a few formal definitions in Appendix A.

\subsection{Bisimulation}

The two games depicted in Fig. 1 are clearly not equivalent when we zoom in on turns and player's available choices. For instance, in $\mathcal{G}_{1}$ there is a choice point where $E$ can decide whether the game ends in $q$ or in $r$, but no such choice point occurs in $\mathcal{G}_{2}$. At this level, a good notion of game equivalence is modal bisimulation, [7]. The two games $\mathcal{G}_{1}, \mathcal{G}_{2}$ are not bisimilar.

This difference also shows concretely in a language that matches the semantic equivalence relation of bisimulation. The propositional modal logic that matches bisimulation can define many properties of our games in detail. So, at this finegrained level of structure, standard modal logic is a good language for games.

\subsection{Power Equivalence}

Next, we abstract away from specific moves and choices, and focus on players' powers for controlling outcomes. Then the two games may be considered the same. In $\mathcal{G}_{1}$, A has a strategy "left" which forces the game to end with outcome $p$, something we can write as the power $\{p\}$. A also has a strategy "right" which forces the set of outcomes $\{q, r\}$. A can do no better than this: the eventual choice between $q$ and $r$ is up to player E. As for the other player, E's strategy "left" forces $\{p, q\}$ (since A can always go left at the start), and E's strategy "right" forces $\{p, r\}$. Now, if we compute powers of the players, we will find the same powers in $\mathcal{G}_{2}$. This is obvious for player $\mathrm{E}$, but since player $\mathrm{A}$ has four strategies now, we need a bit of work. The power $\{p\}$ comes from A's strategy "left, left", the power $\{q, r\}$ from "right, right". The remaining strategies "left right" and "right left" give powers $\{p, r\}$ and $\{q, p\}$, but these can be considered derived, being weakenings of the power $\{p\}$ that A already has. Here we use monotonicity: powers of a player are closed under taking supersets.

This argument is an informal first pass. Formal definitions of the above kind of games and the powers of players in these can be found in Appendix A.

The power level, too, comes with a perspicuous language for describing game structure, viz. a modal logic over neighborhood models whose relations $N^{i} s X$ record that player $i$ has a power $X$ in the relevant game. Naturally, this modal language describes less detail of the extensive game tree. But the power perspective also has another logic connection. The above two game trees mirror a syntax for logical formulas where A controls conjunctions and $E$ disjunctions. And then the equivalence of the above games qua powers reflects the distributive law of propositional 
logic. Indeed, most game semantics for logics operate at the level of powers, often associated with 'winning strategies'.

Much further logical theory for both approaches is developed in [2]. However, as will be shown now, there is yet a third analysis of the question whether the above two games are equivalent, intermediate between the preceding two. Crucially, it pays attention to what both players can achieve interactively.

\subsection{Basic Powers}

Monotonicity is appealing from the perspective of one individual player, but it ignores what others can achieve. Here is how this shows in Example 2.1:

In the game $\mathcal{G}_{2}$, the power $\{p, q\}$ of player $\mathrm{A}$ corresponds to something that can be realized in the following sense: A can decide to narrow down the possible outcomes to $\{p, q\}$, but leave the choice of which of these outcomes to player $\mathrm{E}$. But this is not a power $\mathrm{A}$ has in game $\mathcal{G}_{1}$ : to allow for the outcome $p$, he needs to go "left", but this precludes the possibility that E ever gets to choose $q$. Thus, from a more interactive point of view, the two games are not equivalent. This informal explanation will soon be sharpened up, see Example 2.3.

Powers that allow one payer a restriction and the other player a realisation of each remaining option are the subject of this paper. They refine standard powers that focus on what single players can achieve by themselves to move closer to the game-theoretic notion of an equilibrium, and to social scenarios generally, taking into account how each agent contributes to the overall outcome of a game. Moreover, technically, they are a suggestive pilot for finding interesting game equivalences in between bisimulation and standard power equivalence. ${ }^{1}$

Here is a more precise description. A basic power of player $P$ in an extensive game is a set of outcomes $X$ associated with some strategy $\sigma$ for $\mathrm{P}$ as follows: $X$ is the set of all outcomes at endpoints that can be reached when $\mathrm{P}$ plays according to $\sigma$, while the choices of the other players are free. When the game is infinite, the outcomes meant here are induced by complete histories. To state this more formally, in what follows, $\mathbb{G}(A, O)$ denotes the set of games for players $A$ with outcomes in $O$. For two-player games, the players are named $\mathrm{E}$ and $\mathrm{A}$, where $\overline{\mathrm{E}}=\mathrm{A}$ and $\overline{\mathrm{A}}=\mathrm{E}$. Let $\mathcal{G}$ be any game in $\mathbb{G}(A, O)$, with $a \in A$.

Definition 2.2 A non-empty set $P \subseteq O$ is a basic power for $a$ in $\mathcal{G}$ if there is a strategy $\sigma$ for $a$ in $\mathcal{G}$ such that $P=\{o(m) \mid m \in \operatorname{Match}(\sigma)\}$. The set of all basic powers for a player defined in this way is denoted by $B_{a}(\mathcal{G})$.

\footnotetext{
${ }^{1}$ An alternative perspective on the difference between the two games in our example would bring in imperfect information of players about what the other player has done. We have chosen to pursue the interactive perspective for now, focusing on interactive powers, but a discussion of players' information and knowledge will be found in Sections 8.2 and 9.2.
} 
Here, Match $(\sigma)$ is the set of all complete histories $m$ of $\mathcal{G}$ where $a$ plays according to $\sigma$ at each of her turns, and $o$ is the map provided by $\mathcal{G}$ that assigns outcomes to histories. See Appendix A for details.

The same definition works in extensive games with imperfect information, [18], where nodes are partitioned in equivalence classes ('information sets') for players, and the strategies for a player $i$ satisfy the additional constraint that they assign the same move throughout each information set for $i$. We will use this generalized setting in much of our analysis later on.

Example 2.3 (Example 2.1 revisited with basic powers) The basic powers of player A in game $\mathcal{G}_{1}$ are $\{p\},\{q, r\}$, while those of $\mathrm{E}$ are $\{p, q\},\{p, r\}$. In game $\mathcal{G}_{2}$, the basic powers of $E$ are the same as in game $\mathcal{G}_{1}$, but those of $\mathrm{A}$ are $\{p\},\{p, q\},\{p, r\},\{q, r\}$.

In terms of basic powers then, the two games in Fig. 1 are not the same. Given our general motivation for the interest of basic powers, the preceding discussion suggests a new notion of game equivalence:

Definition 2.4 Two games are said to be basic power equivalent if each player has the same basic powers in both.

In this paper, we study this stricter game equivalence with logical techniques. We modify the theory of power equivalence to obtain new representation theorems, complete game logics in newly designed languages, and algebras of game constructions for basic powers. In the process, we find new themes as well, in particular, a pervasive role for imperfect information and the need for an extended notion of relational rather than functional strategies.

As we noted, basic powers have a game-theoretic slant, moving from the egocentric winning strategies found in much of the logical and computational literature, cf. [2], to 'qualitative equilibria' that depend on what all players do. We will point out further connections with game theory as we go.

\section{Logical Perspectives on Game Equivalence}

Before starting the study of basic powers, we recall some notions and results on standard powers in extensive games viewed as non-empty subsets of a total set of outcomes $\mathcal{O}$, [2]. First, here are three properties of powers of players A, E:

- Non-emptiness: Each player has at least one power.

- Consistency: If $X$ is a power of player A, and $Y$ a power of player $\mathrm{E}$, then $X \cap Y \neq \emptyset$.

- Monotonicity: If $X$ is a power of a player, then so is any superset $Y \supseteq X$.

- Determinacy: If $X$ is not a power of a player, then $\mathcal{O}-X$ is a power of the other one of the players. 
A simple representation result shows that these conditions are characteristic:

Theorem 3.1 The following are equivalent for families $\mathcal{X}, \mathcal{Y}$ of subsets of $\mathcal{O}$ :

1. Consistency, Monotonicity, and Determinacy hold for $\mathcal{X}, \mathcal{Y}$,

2. $\mathcal{X}, \mathcal{Y}$ are the powers of two players in some game of perfect information.

There is also a characterization for the more general case of imperfect information games that carry equivalence relations $\sim$ for both players, giving their uncertainty about where they are in the game tree. Equivalence classes of these relations are often called 'information sets'. It is assumed that -connected points have the same available moves, and importantly, the only allowed strategies are those that assign the same move throughout information sets for the player. Apart from this, the definition of powers is the same as before.

Theorem 3.2 The following are equivalent for families $\mathcal{X}, \mathcal{Y}$ of subsets of $\mathcal{O}$ :

1. Consistency and Monotonicity hold for $\mathcal{X}, \mathcal{Y}$,

2. $\mathcal{X}, \mathcal{Y}$ are the powers of two players in some game of imperfect information.

Now we generalize sameness of powers to allow for comparison across different games. Indeed, going further, we move from game trees altogether to more abstract state spaces connected with games, with abstract relations on states that indicate powers. These are the proper models for our logic to come.

Definition 3.3 Fix a set $\mathrm{Gm}$ of labels for games. A game state model $\mathfrak{M}=(S, N, V)$ has a set of states $S$, a valuation $V$ for proposition letters, and abstract neighborhood relations $N_{\mathrm{P}}^{g} \subseteq S \times \mathcal{P} S$ for each game $g \in \mathrm{Gm}$ and each player $\mathrm{P} \in\{\mathrm{A}, \mathrm{E}\}$. A game state model is a power model if for all games $g$ and $s \in S$, the pair of families $N_{\mathrm{A}}^{g}[s], N_{\mathrm{E}}^{g}[s]$ satisfies Consistency and Monotonicity.

The relation $N_{\mathrm{P}}^{g} s X$ says that, with game $g$ started in state $s$, player $\mathrm{P}$ has a strategy guaranteeing that $g$ will end in some state in $X$ as its outcome. One can view states in $S$ as positions associated with nodes in a game tree, not necessarily one-to-one, as in graph or board games. Much information can be captured at such a level, including invariants that determine players' powers.

Definition 3.4 Let $\mathfrak{M}=(W, N, V), \mathfrak{M}^{\prime}=\left(W^{\prime}, N^{\prime}, V^{\prime}\right)$ be game state models. A binary relation $B \subseteq W \times W^{\prime}$ is a power bisimulation if the following conditions hold for all $s B s^{\prime}$ and each game $g$ and player $\mathrm{P}$ :

Harmony $s, s^{\prime}$ satisfy the same atomic proposition letters.

Forth For all $X$ with $N_{\mathrm{P}}^{g} s X$, there exists an $X^{\prime}$ such that $N_{\mathrm{P}}^{\prime g} s^{\prime} X^{\prime}$ and also:

Forth-Back For all $v^{\prime} \in X^{\prime}$ there is some $v \in X$ such that $v B v^{\prime}$.

Back For all $X^{\prime}$ with $N_{\mathrm{P}}^{\prime g} s^{\prime} X^{\prime}$, there is some $X$ such that $N_{\mathrm{P}}^{g}{ }_{s} X$ and also:

Back-Forth For all $v \in X$ there is some $v^{\prime} \in X^{\prime}$ such that $v B v^{\prime}$. 
States in two pointed game models are power bisimilar, written $\mathfrak{M}, w \leftrightarrows \mathfrak{N}, v$, if there is a power bisimulation $B$ between $\mathfrak{M}$ and $\mathfrak{N}$ such that $w B v$.

There is a modal language matching this level of game description. The formulas of the basic modal logic PL of powers are defined as follows:

$$
\varphi:=p \in \operatorname{Prop}|\varphi \wedge \varphi| \neg \varphi|\langle g, \mathrm{E}\rangle \varphi|\langle g, \mathrm{~A}\rangle \varphi
$$

Semantics of formulas in game state models are defined by the usual recursion, where the clause for the modal operators reads: $\mathfrak{M}, s \Vdash\langle g, \mathrm{P}\rangle \varphi$ iff there is some $X \subseteq S$ with $N_{\mathrm{P}}^{g} s X$ and $\mathfrak{M}, v \Vdash \varphi$ for all $v \in X$. Informally, this says that "player $\mathrm{P}$ can force the condition $\varphi$ to hold for the outcomes of game $g$ ".

Fact 3.5 Formulas of the language of $\mathrm{PL}$ are invariant for power bisimulation.

Fact 3.6 Together with a complete system of axioms for classical propositional logic and the usual rules of modus ponens and uniform substitution, the axioms and rules in Fig. 2 are sound and complete for validity over game state models. Furthermore, $\mathrm{PL}$ is decidable and has the finite model property.

Game state models for perfect information games also satisfy Determinacy, and they are complete by strengthening the axiom Con to an equivalence:

$$
\langle g, \mathrm{P}\rangle p \leftrightarrow \neg\langle g, \overline{\mathrm{P}}\rangle \neg p
$$

One can extend PL with natural game constructions of choices for the two players: $\mathcal{G}_{1} \cup \mathcal{G}_{2}, \mathcal{G}_{1} \cap \mathcal{G}_{2}$, game dual $\mathcal{G}^{d}$ switching roles, and sequential composition $\mathcal{G}_{1} \circ \mathcal{G}_{2}$. These validate a game algebra whose basic part for choice and dual is a 'De Morgan algebra', while $\circ$ is an associative binary operation that is monotonic in both arguments, and distributive over its left, but not its right argument. This algebra can be added in the format of a dynamic logic [19], and the semantic interpretation of this logic on game state models relies on the fact that each of the operations is safe for standard neighborhood bisimulations.

Fact 3.7 The dynamic logic of the operations $\cup, \cap$, o over game state models is decidable and completely axiomatizable.

$\begin{array}{rlr}\text { Con: } \quad\langle g, \mathrm{P}\rangle p \rightarrow \neg\langle g, \overline{\mathrm{P}}\rangle \neg p & \text { NonEm: } \quad\langle g, \mathrm{P}\rangle \top \\ \text { Mon: } & \frac{\varphi \rightarrow \psi}{\langle g, \mathrm{P}\rangle \varphi \rightarrow\langle g, \mathrm{P}\rangle \psi}\end{array}$

Fig. 2 Axioms and rules for the logic PL 
In what follows, we want to preserve the methodology behind these results, and it turns out that we can. But there will be surprises in making things work.

\section{Representation Theorem for Basic Powers}

Our first topic is an abstract characterization of players' basic powers in extensive games with imperfect information, generalizing the approach taken in [1]. We restrict attention to the two-player case, leaving aside the greater subtleties of powers in many-player games, [22]. The analysis to follow mirrors a common construction in the literature on effectivity functions and coalition logics, [20, 21], in its use of 'product spaces' of highly structured moves. Moreover, the set-theoretic conditions to be stated below closely resemble those of [14], which analyzes a more complex setting of coalitional powers.

\subsection{Imperfect Information Games}

We start with basic powers in extensive games of imperfect information. These are defined just as before, but keeping in mind that we only consider uniform strategies for each player. Consider the following three conditions:

- Non-Emptiness: Each player has at least one basic power.

- Consistency: If $X$ is a basic power for player A, and $Y$ is a basic power for player $\mathrm{E}$, then $X \cap Y \neq \emptyset$.

- Exhaustiveness: For each basic power $X$ of player $\mathrm{P}$ and each $x \in X$, there is a basic power $Y$ for player $\overline{\mathrm{P}}$ such that $x \in Y$.

Fact 4.1 Basic powers satisfy Non-Emptiness, Consistency, Exhaustiveness.

A formal proof of this fact can be found in Appendix B. ${ }^{2}$

Our first main result is a representation theorem for basic powers.

Theorem 4.2 The following are equivalent for families $\mathcal{X}, \mathcal{Y}$ of subsets of $\mathcal{O}$ :

1. Non-Emptiness, Consistency and Exhaustiveness hold for $\mathcal{X}, \mathcal{Y}$,

2. $\mathcal{X}, \mathcal{Y}$ are the sets of basic powers of the two players in some game of imperfect information whose possible outcomes are the set $\mathcal{O}$.

Proof One direction is the content of Fact 4.1. For the converse direction from (1) to (2), we define a game $\mathcal{G}$ as follows. $\mathcal{G}$ has two steps: first A makes a move, then $\mathrm{E}$ makes a move. Moreover, $\mathrm{E}$ does not know which move player $\mathrm{A}$ has played: the game $\mathcal{G}$ has imperfect information. The intuitive idea here is that $A$ offers sets of outcomes from his list of basic powers while $E$ then chooses particular outcomes from these. To make this work, some tricks are needed in the precise definition of the game, whose rationale will be explained later on.

\footnotetext{
${ }^{2}$ Incidentally, Non-emptiness and Consistency imply that basic powers are non-empty sets.
} 
Formally, we define an extensive game $\mathcal{G}=(\mathcal{T}, t, o, \Pi)$ as follows, where $T$ stands for the nodes, $t$ is the turn function, $o$ the map assigning outcomes to histories, and $\Pi$ is the partition into information sets. For a start, we set:

M(A) is the set of all triples $(X, i, x)$ with $X \in \mathcal{X}, x \in X$ and $i \in\{0,1\}$

$\mathbf{M}(\mathrm{E})$ is the set of all triples $(Y, j, f)$ such that $Y \in \mathcal{Y}, j \in\{0,1\}$ and $f$ is a choice function from the set $\mathbf{M}(\mathrm{A})$ to the set of outcomes $\mathcal{O}$ picking its value for each triple $t=(X, i, x)$ subject to the following constraints:

1. Always, $f(t) \in X \cap Y$

2. In addition, if $i=j$ and $x \in X \cap Y$, then $f(t)=x$.

Now define $\mathcal{T}$ as the set of all sequences of length at most 2 consisting of at most one object in $\mathbf{M}(\mathrm{A})$ followed by one from $\mathbf{M}(\mathrm{E})$. The turn function $t$ is obvious from this description. For $\Pi$ we pick the partition that has all sequences of length 1 in the same information cell, while consisting of singletons elsewhere. Finally, the outcome function $o$ sends a history $(t,(Y, j, f))$ with $t=(X, i, x)$ to the object $f(t)$ in $\mathcal{O}$ as defined above.

The diagrams in Fig. 3 show the four basic types of situation that may occur at the end of a play according to this definition of the moves. In the first round of the game, Player $\mathrm{A}$ chooses a set $X \in \mathcal{X}$ together with an element $x$ of $X$, plus some $i \in\{0,1\}$. In the second round, Player E chooses a set $Y \in \mathcal{Y}$ together with a function $f$ which picks an element $f(X, i, x)$ of $X \cap Y$, and also chooses some $j \in\{0,1\}$. We make a case distinction depending on whether or not $x \in X \cap Y$; the situation where $x \notin X \cap Y$ is depicted by the diagram $\mathrm{A}$, and the case where $x \in X \cap Y$ is depicted by diagram B. In both cases we draw $x$ as a small empty circle $\circ$ and $f(x)$ as a small filled circle $\bullet$. In situation A the outcome of the play is $f(x)$, but in situation B the outcome depends on whether the players chose the same index, i.e. whether $i=j$. The outcomes in the four possible cases are shown in the matrix displayed to the right in Fig. 3.

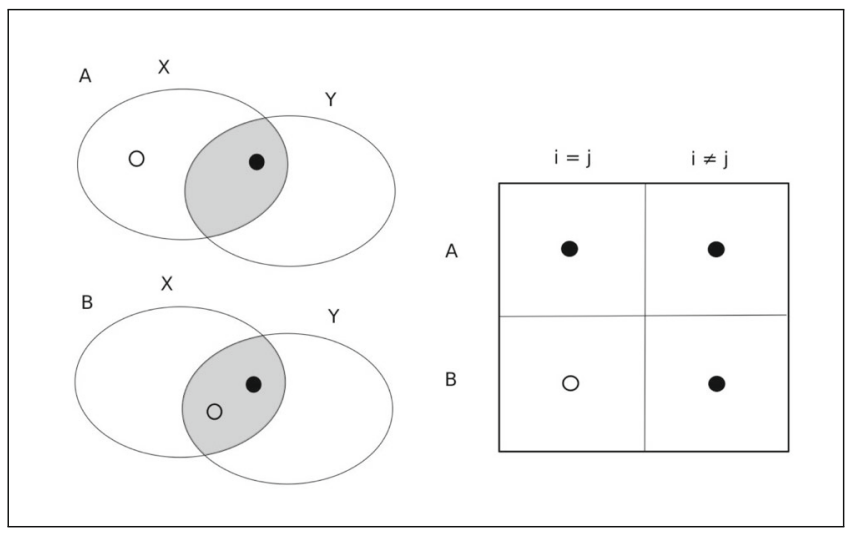

Fig. 3 Choice functions for E's moves 
Note that in this two-step game, the strategies for the first player $A$ are just the moves for A. Because of the imperfect information for the second player $E$, strategies for $\mathrm{E}$ can also be identified with moves, that is, indices corresponding to triples $(Y, j, f)$, since the same choice must be made at each turn.

By Consistency, for any $X \in \mathcal{X}$ and $Y \in \mathcal{Y}$, the intersection $X \cap Y$ is non-empty, which means that the set of choice functions $f$ satisfying conditions 1 and 2 above with respect to any given $X, Y, i, j, x$ is non-empty too. Hence the Non-Emptiness constraint guarantees that both players have non-empty sets of moves at their turns, so we have a well-defined game $\mathcal{G}$ of imperfect information.

The theorem is proved now if we can establish the following:

Claim 4.3 The basic powers of the players in $\mathcal{G}$ are $\mathcal{X}$ for $\mathrm{A}$ and $\mathcal{Y}$ for $\mathrm{E}$.

Proof First we consider the case of player A.

Subclaim 1 The basic power corresponding to the move $(X, i, x)$ is the set $X$.

Proof Note that the set of all outcomes of branches starting with $(X, i, x)$ equals the set of all $f(X, i, x) \in \mathcal{O}$ for all triples $(Y, j, f)$ from $\mathbf{M}(\mathrm{E})$. We first show that only elements from the set $X$ will be chosen. (a) Inclusion $\subseteq$ : By the definition of the functions $f$, they choose their value on $(X, i, x)$ in sets $X \cap Y \subseteq X$.

Next, we must check that each element of $X$ gets chosen. Here the point of our construction becomes clear. The triples are needed in $\mathbf{M}(\mathrm{A})$ to make sure that each object will get chosen sometime. The role of the indices in the triples $(X, i, x),(Y, j, f)$ is revealed as well: they allow choice functions $f$ occurring in $\mathbf{M}(\mathrm{E})$ to still choose other objects in $X$ when the indices do not match.

(b) Inclusion $\supseteq$ : Consider any point $x^{\prime} \in X$. By Exhaustiveness, there is some $Y \in \mathcal{Y}$ with $x^{\prime} \in X \cap Y$. Let $f$ be a choice function defined as follows: pick any $i \in\{0,1\}$, given $(Z, r, z) \in \mathbf{M}(\mathrm{A})$, set $f(Z, r, z)=z$ if $r=i$ and $z \in Y \cap Z$, set $f(Z, r, z)=x^{\prime}$ if $r=|i-1|$ and $x^{\prime} \in Y \cap Z$, and set $f(Z, r, z)$ to some arbitrary element of $Y \cap Z$ otherwise (which is guaranteed to exist by Consistency). Then the triple $(Y,|i-1|, f)$ is a legitimate move for $\mathrm{E}$ according to the above definition, and we have $o((X, i, x),(Y,|i-1|, f))=x^{\prime}$.

Thus, the basic powers of A in $G$ equal the family $\mathcal{X}$. Next, consider player $\mathrm{E}$, recalling that strategies are just the moves, given the imperfect information.

Subclaim 2 For any move $(Y, i, f)$, its corresponding basic power is $Y \in \mathcal{Y}$.

Proof (a) Inclusion $\subseteq$ : By definition, for all histories starting with $(X, i, x)$ and followed by $(Y, j, f)$, the outcome function $o$ assigns the value $f(X, i, x) \in X \cap Y \subseteq Y$. (b) Inclusion $\supseteq$ : We show that all objects $y \in Y$ will in fact be chosen somewhere by the given function $f$. Consider any $y \in Y$. By Exhaustiveness, there is an $X \in \mathcal{X}$ with $y \in X \cap Y$. For that $X,(X, i, y)$ is a possible move in $\mathbf{M}(\mathrm{A})$. By the second constraint on moves for $\mathrm{E}$, we have $o((X, i, y),(Y, i, f))=f(X, i, y)=y$. 
Claim 4.3 now follows from the two Subclaims.

This concludes the proof of the representation theorem for basic powers.

The analysis given here is for two-person games only. The case of three or more persons would seem to involve conditions on basic powers for coalitions of players whose formulation can be delicate, even for the monotonic case, cf. [14]. In line with this, the logic of games with three or more players has additional complexities over the two-player case, $\mathrm{cf}$. $[6,25]$ on cylindric modal logics.

\subsection{Standard Game-Theoretic Angles}

Our approach relates to game theory in various ways. For instance, the proof of Theorem 4.2 shows that arbitrary extensive games with imperfect information are equivalent, qua basic powers, with two-step games. But there is more.

Strategic Forms Our representation theorem also characterizes games in strategic form. To see the connection, we note an analogy between basic powers in game trees and in the rows and columns of two-player matrix games.

Example 4.4 (Strategic form games and basic powers) Revisiting Example 2.1, the strategic forms of the two games are as follows, with rows encoding strategies for $\mathrm{A}$ and columns strategies of $\mathrm{E}$ :

$$
\begin{array}{|l|l|}
\hline p & p \\
\hline q & r \\
\hline
\end{array} \quad \begin{array}{|l|l|}
\hline p & p \\
\hline q & p \\
\hline p & r \\
\hline q & r \\
\hline
\end{array}
$$

Now one can read off the basic powers of the two players directly from the rows and columns, and it is clear that the two games are not basic power equivalent.

Given a two-player game in strategic form, let the yield of a row or column be the unordered set of outcomes appearing in it.

Theorem 4.5 The conditions Non-Emptiness, Consistency and Exhaustiveness are necessary and sufficient for representation of yields of rows and columns in a strategic form two-player game.

Proof This follows directly from the proof of Theorem 4.2. The basic powers of players in our two-step extensive game are identical with the yields of rows and columns in the corresponding strategic normal form game.

Even so, basic power equivalence for games is not the same as having the same strategic form (up to permutation and duplication of rows and columns). 
Example 4.6 (Strategic form equivalence versus basic power equivalence) Both players have the same basic powers in the following games:

$$
\begin{array}{|l|l|l|}
\hline 0 & 1 & 0 \\
\hline 1 & 0 & 0 \\
\hline 0 & 0 & 0 \\
\hline
\end{array} \quad \begin{array}{ll|l|l|}
\hline 1 & 1 & 0 \\
\hline 0 & 0 & 0 \\
\hline
\end{array}
$$

However, there is clearly no way to transform one game into the other simply by duplicating and switching the order of rows and columns. Moreover, viewed as models for a cylindric modal logic $\mathbf{S 5}^{2}$ with the usual "horizontal" and "vertical" accessibility relations, [11], and 1,0 regarded as truth values of some fixed propositional variable, the two games are not related by any modal bisimulation.

Perfect Information Games An important special case are games with perfect information, where all information sets are singletons. In this case, additional properties hold, such as this Determinacy condition for standard powers:

Determinacy' For each set $X \subseteq \mathcal{O}$, either one player has a basic power contained in $X$, or the other player has a basic power contained in $\mathcal{O}-X$.

We have no generalization of our results to powers with perfect information.

Conjecture 4.7 Consistency, Exhaustiveness, and Determinacy' are necessary and sufficient for representing basic powers in games of perfect information.

This result may be harder to prove than the earlier one. For families of sets satisfying Consistency, Monotonicity and Determinacy, one can find a two-step game of perfect information inducing exactly these sets as the standard monotonic powers for the players. But with basic powers, such a simple method does not work. The reason lies in the following observation.

Example 4.8 (Basic powers in perfect information games) Consider the extensive game shown in Fig. 4. Basic powers for $\mathrm{A}$ are $\{p\},\{q, r\},\{q, s\}$, and for $\mathrm{E}:\{p, q\}$, $\{p, r, s\}$. However, it is easy to see that no two-step perfect information game produces just these basic powers.

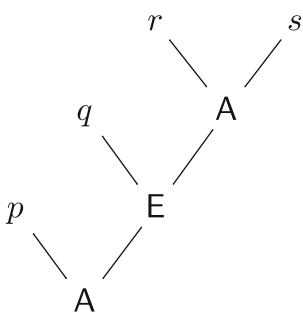

Fig. 4 A three-step game 
Thus even the sophisticated representation style of Theorem 4.2 fails: for basic powers with perfect information, games with more rounds may be essential.

\section{Modal Logic for Basic Powers}

\subsection{Instantial Neighborhood Logic for Basic Powers}

To find a logical perspective on basic powers, note that basic power equivalence can be generalized to game state models as before. Again, the invariance issue then shifts to: When are two game state models the same at the level of basic powers? The answer is in the following definition.

Definition 5.1 Let $\mathfrak{M}=(W, N, V), \mathfrak{M}^{\prime}=\left(W^{\prime}, N^{\prime}, V^{\prime}\right)$ be game state models. The relation $B \subseteq W \times W^{\prime}$ is a basic power bisimulation if the following conditions hold for all $s B s^{\prime}$ and each game $g$ and player $\mathrm{P}$ :

Harmony $\quad s, s^{\prime}$ satisfy the same atomic proposition letters.

Forth For all $X$ with $N_{\mathrm{P}}^{g} s X$, there is some $X^{\prime}$ such that $N_{\mathrm{P}}^{\prime g} s^{\prime} X^{\prime}$ and also:

Forth-Forth For all $v \in X$, there is a $v^{\prime} \in X^{\prime}$ such that $v B v^{\prime}$.

Forth-Back For all $v^{\prime} \in X^{\prime}$, there is a $v \in X$ such that $v B v^{\prime}$.

Back For all $X^{\prime}$ with $N_{\mathrm{P}}^{\prime g} s^{\prime} X^{\prime}$, there is some $X$ such that $N_{\mathrm{P}}^{g} s X$ and also:

Back-Forth For all $v \in X$, there is a $v^{\prime} \in X^{\prime}$ such that $v B v^{\prime}$.

Back-Back For all $v^{\prime} \in X^{\prime}$, there is a $v \in X$ such that $v B v^{\prime}$.

We say that two states in two given pointed game state models are basic power bisimilar, written $\mathfrak{M}, w \leftrightarrows \mathfrak{N}, v$, if there is a basic power bisimulation $B$ between $\mathfrak{M}$ and $\mathfrak{N}$ such that $w B v$.

Next, the transition to logic follows a familiar pattern. Semantic equivalence relations between models induce formal languages for describing invariant properties. What remains to be done here is to identify a natural formalism for game description that matches basic power bisimulation.

The language that serves this purpose is a richer version of the standard modal languages for neighborhood models that have been used in the literature on players and coalitions in games and related social scenarios. It was introduced under the name instantial neighborhood logic in [4]. We denote it here by BPL, for "basic power logic". Its formulas are defined by the following grammar:

$$
\varphi:=p \in \operatorname{Prop}|\varphi \wedge \varphi| \neg \varphi \mid\langle g, \mathrm{P}\rangle(\Psi, \varphi)
$$

where $\Psi$ is a finite set of BPL-formulas, called the instantial formulas. In particular, the modalities $\langle g, \mathrm{P}\rangle$ are indexed here for a game $g$ and a player $\mathrm{P}$. 
As a notational convention, if $\Psi=\left\{\psi_{1}, \ldots, \psi_{n}\right\}$, we will often write the modality as $\langle g, \mathrm{P}\rangle\left(\psi_{1}, \ldots, \psi_{n}, \varphi\right)$ rather than $\langle g, \mathrm{P}\rangle(\Psi, \varphi)$. However, $\psi_{1}, \ldots, \psi_{n}$ should then still be understood as a set rather than a tuple of formulas.

That this is in fact the right modal language for basic powers was observed, although in different terminology, in [4], where the following results were proved:

Fact 5.2 All formulas of BPL are invariant for basic power bisimulations.

Fact 5.3 (Hennessy-Milner property) Two finite, pointed game state models are basic power bisimilar if, and only if, they satisfy the same formulas in BPL.

\section{Axiomatization}

In this section we axiomatize the valid formulas of BPL, thus pinning down the modal logic of basic powers. Our system is a gentle modification of instantial neighborhood logic, and the key step of the completeness proof is to verify that the conditions characterizing basic powers can be captured by suitable axioms.

As axioms and rules for BPL, we take all propositional tautologies, modus ponens, uniform substitution plus the principles displayed in Figs. 5 and 6:

Theorem 6.1 The displayed axioms and rules for BPL are sound and complete.

Proof Soundness requires a check of the axioms. For the INL part (Fig. 5), these are mostly obvious, except for Lem. This says that any property $q$ is either instantiated in the given neighborhood, or its negation holds throughout that neighborhood, justifying a move of $\neg q$ into the universal condition on the right-hand side. This axiom is crucial to our later use of normal forms, cf. [4].

As for the axioms of BPL (Fig. 6), these reflect the three conditions in our representation result that we have already shown to hold for basic powers.

Weak: $\quad\langle g, \mathrm{P}\rangle(\Phi ; p) \rightarrow\langle g, \mathrm{P}\rangle\left(\Phi^{\prime} ; p\right) \quad\left(\Phi^{\prime} \subseteq \Phi\right)$
Un: $\quad\langle g, \mathrm{P}\rangle\left(q_{1}, \ldots, q_{n} ; p\right) \rightarrow\langle g, \mathrm{P}\rangle\left(q_{1} \wedge p, \ldots, q_{n} \wedge p ; p\right)$
Lem: $\quad\langle g, \mathrm{P}\rangle(\Phi ; p) \rightarrow\langle g, \mathrm{P}\rangle(\Phi \cup\{q\} ; p) \vee\langle g, \mathrm{P}\rangle(\Phi ; \neg q \wedge p)$
Bot: $\quad \neg\langle g, \mathrm{P}\rangle(\perp ; p)$
Mon: $\frac{p \rightarrow p^{\prime} \quad q_{i} \rightarrow q_{i}{ }^{\prime} \quad(1 \leq i \leq n)}{\langle g, \mathrm{P}\rangle\left(q_{1}, \ldots, q_{n} ; p\right) \rightarrow\langle g, \mathrm{P}\rangle\left(q_{1}^{\prime}, \ldots, q_{n}^{\prime} ; p^{\prime}\right)}$

Fig. 5 Axioms and rules for instantial neighborhood logic 


\begin{tabular}{rr|}
\hline Non-Em: $\quad\langle g, \mathrm{P}\rangle \top \quad$ Cons: $\quad\langle g, \mathrm{P}\rangle p \rightarrow \neg\langle g, \overline{\mathrm{P}}\rangle \neg p$ \\
Inst: $\quad\langle g, \mathrm{P}\rangle(p ; \top) \leftrightarrow\langle g, \overline{\mathrm{P}}\rangle(p ; \top)$
\end{tabular}

Fig. 6 The additional axioms for BPL

The completeness proof is a normal form argument using finite state descriptions in the style of [4], but the adaptation to basic powers is not trivial, as we must deal with the new frame constraints of Non-emptiness, Consistency and Exhaustiveness. The model construction to follow satisfies these constraints.

More in detail, the completeness proof proceeds in the following steps.

Definition 6.2 The modal depth of a formula is defined inductively by:

$$
\begin{aligned}
& d(p)=0, \quad d(\neg \varphi)=d(\varphi), \quad d(\varphi \wedge \psi)=\max (d(\varphi), d(\psi)), \\
& d(\langle g, \mathrm{P}\rangle(\Gamma ; \varphi))=\max (d[\Gamma \cup\{\varphi\}])+1 .
\end{aligned}
$$

Definition 6.3 Given a finite set of propositional variables $Q$, a BPL formula $\varphi$ is a $Q$-formula if all propositional variables appearing in $\varphi$ belong to $Q$.

Given $k \in \omega$ and a finite set $Q$ of propositional variables, a $(Q, k)$-description is a consistent $Q$-formula $\varphi$ of modal depth $\leq k$, such that for any $Q$-formula $\theta$ of depth $\leq k$, we have $\varphi \vdash \theta$ or $\varphi \vdash \neg \theta$.

By a standard argument, there are at most finitely many $Q$-formulas of depth $\leq k$ up to logical equivalence, given that $Q$ is finite.

A key lemma for the completeness proof is the following result, of a type wellknown in the literature on modal normal forms - cf. [4] for details:

Lemma 6.4 Let $\langle g, \mathrm{P}\rangle(\Gamma ; \varphi)$ be a formula with $\max (d[\Gamma \cup\{\varphi\}]) \leq k$ and $Q$ a finite set of propositional variables containing all variables appearing in this formula. Then $\langle g, \mathrm{P}\rangle(\Gamma ; \varphi)$ is provably equivalent to a disjunction of the form:

$$
\bigvee_{i \in I}\langle g, \mathrm{P}\rangle\left(\Theta_{i} ; \bigvee \Theta_{i}\right)
$$

with I finite, and for each $i \in I, \Theta_{i}$ a finite set of $(Q, k)$-descriptions such that:

- every member of $\Theta_{i}$ provably entails $\varphi$, and

- every member of $\Gamma$ is provably entailed by some member of $\Theta_{i}$.

Fix a finite set of propositional variables $Q$. Given a $Q$-formula $\varphi, \widehat{\varphi}$ is its equivalence class under provable equivalence. For a finite set of formulas $\Gamma$, set

$$
\widehat{\Gamma}=\{\widehat{\varphi} \mid \varphi \in \Gamma\}
$$

We now construct a canonical neighborhood model using our normal forms. 
Definition 6.5 The canonical model $\mathfrak{M}=(W, N, V)$ is defined as follows:

- $\quad W=\{(\widehat{\varphi}, k) \mid \varphi$ is a $(Q, k)$-description and $k<\omega\}$

- For any player $\mathrm{P}$, let $N_{\mathrm{P}}$ be the union of the sets

$$
\{((\widehat{\varphi}, k+1), \widehat{\Gamma} \times\{k\}) \in W \times \mathcal{P}(W) \mid \varphi \vdash\langle g, \mathrm{P}\rangle(\Gamma ; \bigvee \Gamma)\}
$$

and

$$
\{((\widehat{\varphi}, 0), W) \mid(\widehat{\varphi}, 0) \in W\}
$$

- Finally, for any propositional variable $p$, set $V(p)=\{\widehat{\varphi} \mid \varphi \vdash p\}$ if $p \in Q$, $V(p)=\emptyset$ otherwise.

Note that this is well defined, i.e., whether $(\widehat{\varphi}, \widehat{\Gamma}) \in N_{\mathrm{P}}$ is independent of the choice of witnesses $\varphi, \Gamma$ for the equivalence classes.

Lemma 6.6 (Truth lemma) Let $\mathfrak{M}$ be constructed as above, and let $\psi$ be any basic formula of modal depth $\leq k$ whose propositional variables all belong to $Q$, and which is such that all game terms appearing in $\psi$ belong to $\tau$. Then for every $(Q, \tau, k)$-description $\varphi$, we have:

$$
\mathfrak{M},(\widehat{\varphi}, k) \Vdash \psi \text { iff } \varphi \vdash \psi
$$

Proof By induction on $k$. In the case of $k=0, \psi$ is a formula of classical propositional logic and the result follows easily using the definition of the valuation $V$ since we have included all propositional tautologies as axioms.

Assuming that the induction hypothesis holds for $k$, consider a formula $\psi$ of modal depth $\leq k+1$ and let $(\widehat{\theta}, k+1) \in W$. We wish to show that:

$$
\mathfrak{M},(\widehat{\theta}, k+1) \Vdash \psi \text { iff } \theta \vdash \psi
$$

We reason by a sub-induction on the complexity of subformulas of $\psi$, where we consider both subformulas of the form $\langle g, \mathrm{P}\rangle(\Psi ; \varphi)$ and propositional variables not in the scope of any modal operator as the atomic cases.

Since the induction steps for boolean connectives are trivial, and we have already dealt with the case of purely propositional formulas, we focus on the atomic case of a modal formula $\langle g, \mathrm{P}\rangle(\Psi ; \varphi)$. Since $\psi$ was of modal depth $\leq k+1$, every formula in $\Psi \cup\{\varphi\}$ must be of modal depth $\leq k$, so by the main induction hypothesis we get that $\mathfrak{M},(\widehat{\rho}, k) \Vdash \gamma$ iff $\rho \vdash \gamma$, for all $\gamma \in \Psi \cup\{\varphi\}$ and all $(\rho, k) \in W$ such that $\rho$ is a $(Q, \tau, k)$-description.

Suppose that $\mathfrak{M},(\widehat{\theta}, k+1) \Vdash\langle g, \mathrm{P}\rangle(\Psi ; \varphi)$. Then there is a neighborhood of $(\theta, k+1)$ w.r.t. the neighborhood relation $N_{\mathrm{P}}$ of the form $\widehat{\Gamma} \times\{k\}$, such that $\Gamma$ is a set of $(Q, \tau, k)$-descriptions and:

- $\mathfrak{M},(\widehat{\rho}, k) \Vdash \varphi$ for all $\rho \in \Gamma$,

- $\quad$ for all $\delta \in \Psi$ there is some $\rho \in \Gamma$ such that $\mathfrak{M},(\widehat{\rho}, k) \Vdash \delta$.

Hence by the induction hypothesis we get:

- $\rho \vdash \varphi$ for all $\rho \in \Gamma$,

- for all $\delta \in \Psi$ there is some $\rho \in \Gamma$ such that $\rho \vdash \delta$. 
It follows that $\bigvee \Gamma \vdash \varphi$. By definition of the neighborhood relation $N_{\mathrm{P}}$ we have $\theta \vdash\langle g, \mathrm{P}\rangle(\Gamma ; \bigvee \Gamma)$. But due to the rule (Mon) and the axiom (Weak) we get $\langle g, \mathrm{P}\rangle(\Gamma ; \bigvee \Gamma) \vdash\langle g, \mathrm{P}\rangle(\Psi ; \varphi)$, hence $\theta \vdash\langle g, \mathrm{P}\rangle(\Psi ; \varphi)$ as required.

Conversely, suppose $\theta \vdash\langle g, \mathrm{P}\rangle(\Psi ; \varphi)$. Since $\theta$ is a $(Q, \tau, k+1)$-description, we must have $\theta \vdash\langle g, \mathrm{P}\rangle(\Gamma ; \bigvee \Gamma)$ for some disjunct $\langle g, \mathrm{P}\rangle(\Gamma ; \bigvee \Gamma)$ of the normal form of $\langle g, \mathrm{P}\rangle(\Psi ; \varphi)$ given by Lemma 6.4 . Hence $((\widehat{\theta}, k+1), \widehat{\Gamma} \times\{k\}) \in N_{\mathrm{P}}$. We recall that by Lemma 6.4 the set $\Gamma$ is a set of $(Q, \tau, k)$-descriptions such that:

- $\rho \vdash \varphi$ for all $\rho \in \Gamma$,

- for all $\delta \in \Psi$ there is some $\rho \in \Gamma$ such that $\rho \vdash \delta$.

By the induction hypothesis we get:

- $\mathfrak{M},(\widehat{\rho}, k) \Vdash \varphi$ for all $\rho \in \Gamma$,

- for all $\delta \in \Psi$ there is some $\rho \in \Gamma$ such that $\mathfrak{M},(\widehat{\rho}, k) \Vdash \delta$.

Hence $\mathfrak{M},(\widehat{\theta}, k+1) \Vdash\langle g, \mathrm{P}\rangle(\Psi ; \varphi)$ as required.

The addition we need for present purposes is the following lemma:

Lemma 6.7 The structure $\mathfrak{M}$ constructed above is a power model, that is, it satisfies the Non-emptiness, Consistency and Exhaustiveness constraints.

Proof First, note that all the conditions hold for the image of each relation on an element of $W$ of the form $(\widehat{\varphi}, 0)$. So we can focus on the images of relations of the form $N_{\mathrm{P}}$ on states of the form $(\widehat{\varphi}, k+1)$ for some $k$.

The Non-emptiness condition is straightforward from the axiom (Non-Em) of BPL. Next, for Instantiatedness, suppose that

$$
((\widehat{\varphi}, k+1), \widehat{\Theta} \times\{k\}) \in N_{\mathrm{A}}
$$

By definition, we get $\varphi \vdash\langle g, \mathrm{E}\rangle(\Theta ; \bigvee \Theta)$. Pick an element $(\widehat{\theta}, k) \in \Theta \times\{k\}$. By (Weak) and (Mon) we get $\langle g, \mathrm{E}\rangle(\Theta ; \bigvee \Theta) \vdash\langle g, \mathrm{E}\rangle(\theta ; \top)$, so $\varphi \vdash\langle g, \mathrm{E}\rangle(\theta ; \top)$. By the axiom (Inst) we get $\varphi \vdash\langle g, \mathrm{~A}\rangle(\theta ; \top)$ as well. Since $\varphi$ is a $(Q, k+1)$-description, it follows from Lemma 6.4 that there is a set $\Psi$ of $(Q, k)$-descriptions with $\varphi \vdash$ $\langle g, \mathrm{~A}\rangle(\Psi ; \bigvee \Psi)$ and such that there exists some $\psi \in \Psi$ with $\psi \vdash \theta$. But since $\psi, \theta$ are both $(Q, k)$-descriptions, this means that $\widehat{\theta}=\widehat{\psi}$, so $(\widehat{\theta}, k)=(\widehat{\psi}, k)$. But then we get $((\widehat{\varphi}, k+1), \widehat{\Psi} \times\{k\}) \in N_{\mathrm{E}}$ and $(\widehat{\theta}, k) \in \widehat{\Psi} \times\{k\}$ as required. The converse direction is proved in the same manner.

For Consistency, let $((\widehat{\varphi}, k+1), \widehat{\Theta} \times\{k\}) \in N_{\mathrm{A}}$ and $\left((\widehat{\varphi}, k+1), \widehat{\Theta}^{\prime} \times\{k\}\right) \in N_{\mathrm{E}}$. Clearly, since $\Theta$ and $\Theta^{\prime}$ are both sets of $(Q, k)$-descriptions, that $\widehat{\Theta} \times\{k\}$ does not intersect $\widehat{\Theta}^{\prime} \times\{k\}$, and so $\bigvee \Theta^{\prime} \rightarrow \neg \bigvee \Theta$. But we have $\varphi \vdash\langle g, \mathrm{E}\rangle(\Theta ; \bigvee \Theta)$, hence $\varphi \vdash\langle g, \mathrm{E}\rangle \bigvee \Theta$ by the axiom schema (Weak). Furthermore we have:

$$
\varphi \vdash\langle g, \mathrm{~A}\rangle\left(\Theta^{\prime} ; \bigvee \Theta^{\prime}\right) \vdash\langle g, \mathrm{~A}\rangle \bigvee \Theta^{\prime} \vdash\langle g, \mathrm{~A}\rangle \neg \bigvee \Theta
$$

But then $\varphi \vdash\langle g, \mathrm{E}\rangle \bigvee \Theta \wedge\langle g, \mathrm{~A}\rangle \neg \bigvee \Theta$, and it follows from the axiom schema (Cons) that the formula $\varphi$ cannot be consistent, which contradicts our assumption that $\varphi$ was a $(Q, k+1)$-description. 
Combining Lemmas 6.7 and 6.6 with the easy observation that any consistent basic formula of depth $\leq k$, variables in $Q$ and atomic games among $\tau$ is provably entailed by some $(Q, k)$-description, ${ }^{3}$ we obtain Theorem 6.1 .

As a corollary to this proof, we get a further property of our new game logic:

Theorem 6.8 BPL is decidable and has the effective finite model property.

\subsection{Special Cases and Variations}

Logics of Specific Games Our move to abstract game state models has one consequence that should be noted. In the original example motivating this paper, powers were sets of endpoints of some concrete game. In that special case, iterating power modalities is almost trivial, as powers of players at endpoints $s$ are just the singleton sets $\{s\}$. But because of this, the logic of game trees will validate further principles that were not in our BPL logic of games.

Fact 6.9 The formula $[i]([i] \varphi \leftrightarrow \varphi)$ is valid on extensive games.

This trivializes iterated play of the current game, while in a general game logic, we can play a game to its end in some state $s$ in the state space, and then restart the game from there. We leave axiomatizating concrete games as an open problem, but we conjecture that adding the preceding principle suffices.

Intermediate Powers Strategies in game trees are not just directed toward endpoints: players can also make sure that play has to pass through relevant intermediate positions. Accordingly, we can define powers that allow both intermediate nodes and endpoints as outcomes. (Cf. the intermediate forcing in Grossi and Turrini [15].) The earlier modal neighborhood language can still describe such powers, but now it gets closer to a standard modal action language over game trees, as powers can also be singletons of states reached after one move. We leave the logic of this game equivalence as one more open problem.

\section{Algebra of Game Constructions}

Our modal neighborhood language describes the structure of games from an "internal" perspective, where formulas are evaluated at a given point in the associated state space of a game. But we can also describe game structure "externally" by means of an algebra of game constructions.

This approach, too, has connections with logic. We start by considering some natural operations on games, and then develop this perspective with games of imperfect information. This may be surprising, as these games have no compositional tree-like

\footnotetext{
${ }^{3}$ This follows in a standard way from Lindenbaum's lemma applied to at most finitely many formulas of depth $\leq k$, variables in $Q$, and game terms among $\tau$, up to provable equivalence.
} 
structure in general (epistemic uncertainty links can cross between subtrees), but we shall see that we can still come a long way.

\subsection{Propositional Operations}

We will use some basic concepts of universal algebra, see for example [9]. For simplicity, we restrict attention to finite games, so that $\mathbb{G}(\{\mathrm{A}, \mathrm{E}\}, O)$ is now the set of finite games with outcomes in $O$. Thus the outcome map of a game $\mathcal{G}$ can be viewed a map $o$ from the leaves in $\mathcal{G}$ into $O$.

Consider a family of extensive games on a fixed set of outcomes $O$. We define operations in a standard manner, with binary $U, \cap$ corresponding to choice for $A, E$ respectively, and a unary operation - for game dual ('role switch').

Definition 7.1 Let $\mathcal{G}_{1}=\left(\mathcal{T}_{1}, t_{1}, o_{1}, \Pi_{1}\right)$ and $\mathcal{G}_{2}=\left(\mathcal{T}_{2}, t_{2}, o_{2}, \Pi_{2}\right)$. The choice games $\mathcal{G}_{1} \cup \mathcal{G}_{2}\left(\mathcal{G}_{1} \cap \mathcal{G}_{2}\right)$ are defined as follows. We first construct a tree $\mathcal{T}^{\prime}$ by adding a new root $r$ with two successors, where the left successor is the root of a subtree isomorphic with $\mathcal{T}_{1}$ via a fixed isomorphism $i_{1}$, and the right successor the root of a subtree isomorphic with $\mathcal{T}_{2}$ via a fixed $i_{2}$. The turn function $t^{\prime}$ is defined by setting $t^{\prime}(r)=\mathrm{A}\left(t^{\prime}(r)=\mathrm{E}\right)$. For a node $u$ in the subtree for the left successor of the root $r$ we set $t^{\prime}(u)=t_{1}\left(i_{1}(u)\right)$, and for a node $u$ in the subtree for the right successor of $r$, we set $t^{\prime}(u)=t_{2}\left(i_{2}(u)\right)$. The outcome map $o^{\prime}$ is defined by setting $o^{\prime}(l)=o_{1}\left(i_{1}(l)\right)$ for a leaf in the subtree corresponding to the left successor of $r$, and $o^{\prime}(l)=o_{2}\left(i_{2}(l)\right)$ for a leaf in the subtree corresponding to the right successor of $r$. We define a partition $\Pi^{\prime}$ by setting

$$
\Pi^{\prime}=\{\{r\}\} \cup\left\{i_{1}^{-1}[Z] \mid Z \in \Pi_{1}\right\} \cup\left\{i_{2}^{-1}[Z] \mid Z \in \Pi_{2}\right\} .
$$

The game $\mathcal{G}_{1} \cup \mathcal{G}_{2}\left(\mathcal{G}_{1} \cap \mathcal{G}_{2}\right)$ is then defined as $\left(\mathcal{T}^{\prime}, t^{\prime}, o^{\prime}, \Pi^{\prime}\right)$.

The definition of $-\mathcal{G}$ is much simpler, it merely changes the turn assignment by switching players at each position, otherwise keeping everything the same.

Definition 7.2 Let $\mathcal{G}=(\mathcal{T}, t, o, \Pi)$. Then $-\mathcal{G}$ is defined to be the structure $\left(\mathcal{T}, t^{-}, o, \Pi\right)$, where $t^{-}$is defined by $t^{-}(u)=\overline{t(u)}$ for each $u \in \mathcal{T}$.

Remark 7.3 These operations make strong assumptions. In particular, the imperfect information stays inside the games connected by $\cup, \cap$, since the initial choice introduces no uncertainty (in other words, the first round of a choice game has perfect information). We will return to this issue later on.

Basic powers in composite games can be straightforwardly computed from the basic powers of their components, as is shown by the following fact:

Fact 7.4 Basic powers of players in these games obey the following conditions:

1. Basic powers for $\mathrm{A}$ in $\mathcal{G}_{1} \cup \mathcal{G}_{2}$ are all of $\mathrm{A}$ 's basic powers in the game $\mathcal{G}_{1}$ plus all of A's basic powers in $\mathcal{G}_{2}$. 
2. Basic powers of $\mathrm{E}$ in $\mathcal{G}_{1} \cup \mathcal{G}_{2}$ are set-theoretic unions of pairs of basic powers of $\mathrm{E}$ in the games $\mathcal{G}_{1}, \mathcal{G}_{2}$ separately.

3. Analogously for powers of the two players in the dual choice game $\mathcal{G}_{1} \cap \mathcal{G}_{2}$.

4. Basic powers of $\mathrm{E}$ in $-\mathcal{G}$ are basic powers of $\mathrm{A}$ in $\mathcal{G}$, and likewise for $\mathrm{A}$.

Proof By a simple verification.

As a direct corollary we get the following proposition, which opens the door to a game algebra of basic power equivalence that can be seen as a counterpart to the well-studied algebra of monotonic power equivalence in $[13,26]$ :

Proposition 7.5 Basic power equivalence is a congruence on the algebra:

$$
\langle\mathbb{G}(\{\mathrm{A}, \mathrm{E}\}, O), \cup, \cap,-\rangle .
$$

Definition 7.6 The game algebra of basic power equivalence $\mathfrak{G}$ (with outcomes $O$ ) is defined to be the quotient:

$$
\langle\mathbb{G}(\{\mathrm{A}, \mathrm{E}\}, O), \cup, \cap,-\rangle / \simeq
$$

An algebraic equation in the signature $\langle\cup, \cap,-\rangle\rangle$ is said to be valid on $\mathfrak{G}$ if it belongs to the equational theory of the algebra $\mathfrak{G}$.

The game algebra of basic powers differs in interesting ways from that for monotonic powers. Before showing this, we generalize, as we did with the logic for basic powers, from extensive game trees to arbitrary game state models $\mathfrak{M}=(S, N, V)$, where the neighborhood relations $N^{i, G} S X$ satisfy abstract analogues of the preceding observations, for all game terms $G$. In doing so, we take a dynamic view of games as state-transforming processes in the style of dynamic game logic (cf. [2, 19, 24]). This leads to an extended algebraic perspective.

Definition 7.7 A dynamic two-player game over a set $X$ (of "states") is a map $g$ : $X \rightarrow \mathbb{G}(\{\mathrm{A}, \mathrm{E}\}, X)$, assigning a game with outcome set $X$ to each state in $X$. We denote the set of dynamic two-player games over $X$ by $\mathbb{D}(\{\mathrm{A}, \mathrm{E}\}, X)$.

The operations $\cup, \cap$ and - are naturally lifted to dynamic games in a componentwise manner, and so is the relation $\simeq$ of basic power equivalence. The following observation shows we get an algebra once more:

Proposition 7.8 Basic power equivalence is a congruence on the algebra:

$$
\langle\mathbb{D}(\{\mathrm{A}, \mathrm{E}\}, O), \cup, \cap,-\rangle .
$$

Definition 7.9 The dynamic game algebra of basic power equivalence $\mathfrak{D}$ (with outcomes $O$ ) is defined to be the quotient:

$$
\langle\mathbb{D}(\{\mathrm{A}, \mathrm{E}\}, O), \cup, \cap,-\rangle / \simeq
$$


Equational validity for $\mathfrak{D}$ is again defined in the standard manner.

The following observations about valid equations are easy to verify.

Fact 7.10 The following are valid in the game algebra of basic powers:

$$
\begin{array}{ll}
x \cup y=y \cup x & x \cap y=y \cap x \\
x \cup(y \cup z)=(x \cup y) \cup z & x \cap(y \cap z)=(x \cap y) \cap z \\
--x=x & \\
-(x \cup y)=-x \cap-y & -(x \cap y)=-x \cup-y .
\end{array}
$$

These are all valid in propositional logic. But there are also striking failures:

Fact 7.11 The following equations are not valid in the algebra of basic powers:

$$
\begin{array}{ll}
x \cup x=x & x \cap x=x \\
x \cup(y \cap z)=(x \cup y) \cap(x \cup z), & x \cap(y \cup z)=(x \cap y) \cup(x \cap z) .
\end{array}
$$

That distribution fails was observed already in our introduction. We only retain a weaker principle which follows from the above valid equations:

$$
x \cup(y \cap x)=(x \cup y) \cap x
$$

Most striking, perhaps, is the failure of the idempotence laws in our algebra:

Example 7.12 (Failure of idempotence) Consider a game $\mathcal{G}$ with outcomes in $O=$ $\{p, q\}$, where Player E moves first, and gets a choice between outcomes $p$ or $q$, with no moves at all for Player A. The basic powers of Player $E$ in $\mathcal{G}$ are just $\{p\}$ and $\{q\}$. But in the game $\mathcal{G} \cap \mathcal{G}$, starting with a choice between two copies of $\mathcal{G}$ for Player $\mathrm{A}$, Player $\mathrm{E}$ clearly also has the basic power $\{p, q\}$, so it is not the case that $\mathcal{G} \cap \mathcal{G} \simeq \mathcal{G}$. The two games are displayed in Fig. 7, with $\mathcal{G}$ to the left, $\mathcal{G} \cap \mathcal{G}$ to the right.

This makes our game algebra a weak propositional logic sharing a failure of distribution with quantum logic, and a failure of idempotence with linear logic.

Open problem 7.13 Axiomatize the complete game algebra of basic powers.

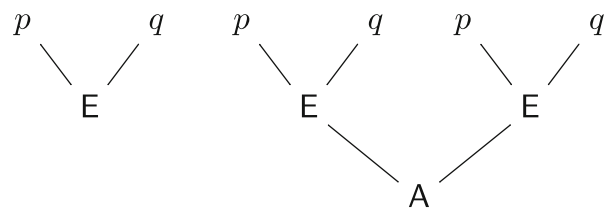

Fig. 7 Failure of idempotence 
An interesting feature of this game algebra is its use as a tool for studying game equivalence under basic powers. Valid laws of propositional logic that interchange conjunctions and disjunctions correspond to transformations that change order of turns in games. Normal forms yield normal forms for games: say, a distributive normal form shows that we can let one player start (all consecutive moves can then be collected into one), then the other, and let the game end there. However, our weaker game algebra for basic powers does not support standard propositional normal forms, a topic to which we return below.

\subsection{Composition and Relational Strategies}

There are further fundamental operations on games. Clearly, games compose to form sequential games, and the corresponding operation is as follows.

Definition 7.14 Given dynamic games $\mathcal{G}_{1}, \mathcal{G}_{2}: X \rightarrow \mathbb{G}(\{\mathrm{A}, \mathrm{E}\}, X)$, we can define the sequential composition $\mathcal{G}_{1} \circ \mathcal{G}_{2}$ by letting $\mathcal{G}_{1} \circ \mathcal{G}_{2}(u)$ be constructed by replacing each leaf $l$ in $\mathcal{G}_{1}(u)$ by a copy of the game tree $\mathcal{G}_{2}\left(o_{1}(l)\right)$, where $o_{1}$ is the outcome map associated with $\mathcal{G}_{1}(u)$.

Remark 7.15 This is just one definition of sequential game composition, as it does not allow non-trivial imperfect information links across sequential games. But for instance, if endpoints of $G$ can have epistemic links, we might want to let this continue to the games placed at these. On the other hand game theory often assumes a mysterious act of "revelation" at the end of the game.

However, there is a more immediate problem to deal with, even for this simple notion. It turns out that basic powers are an inherently "global" property of games, and fail to be compositional with respect to sequential composition.

Fact 7.16 Basic power equivalence is not generally a congruence with respect to the operation of sequential composition on the algebra $\mathbb{D}(\{\mathrm{A}, \mathrm{E}\}, O)$.

Proof Let $O=\{p, q\}$. The two perfect information games displayed in Fig. 8 can clearly be split up as sequential compositions of pairwise basic power equivalent games. But the games are not basic power equivalent themselves, as player $E$ has a basic power $\{p, q\}$ in the game to the right, but not to the left.

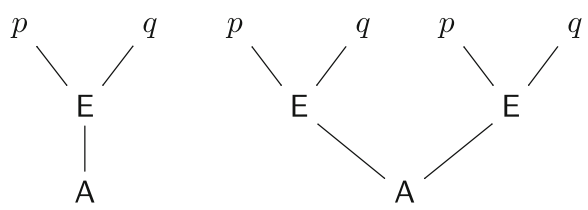

Fig. 8 Failure of safety 
Why does this happen? An obvious culprit is the functional nature of strategies. If a model gets inflated modulo bisimulation, creating copies of earlier moves, then new functions can create new basic powers that were not there before. One solution, then, is to change the functional nature of strategies.

To remedy this situation, we will now widen the notion of a strategy to allow nondeterminism, so that strategies may constrain the moves of a player, but not determine them uniquely. This move is not altogether new: mixed strategies in game theory can be interpreted in a relational way - and the use of relations rather than functions has also been defended for the broader notion of a 'plan' in [2] leading to a better model theory of strategies.

Definition 7.17 A relational strategy for player $\mathrm{P}$ in a game $\mathcal{G}=(\mathcal{T}, t, o, \Pi)$ is a binary relation $\sigma$ over $\mathcal{T}$ such that:

- $\sigma[u] \neq \emptyset$ whenever $u \in t^{-1}[\mathrm{P}]$, and

- $\sigma[u]=\sigma[v]$ if $u, v$ are in the same partition cell in $\Pi$.

The set Match $(\sigma)$ of non-deterministic matches guided by a strategy $\sigma$ is defined in the obvious way. We say that $P \subseteq O$ is a relational basic power of $\mathrm{P}$ if there is a relational strategy $\sigma$ for $\mathrm{P}$ in $\mathcal{G}$ such that $P=\{o(m) \mid m \in \operatorname{Match}(\sigma)\}$. The set of relational basic powers of $\mathrm{P}$ is denoted by $R_{\mathrm{P}}(\mathcal{G})$. We say that $\mathcal{G}_{1}, \mathcal{G}_{2}$ are relational basic power equivalent if $R_{\mathrm{P}}\left(\mathcal{G}_{1}\right)=R_{\mathrm{P}}\left(\mathcal{G}_{2}\right)$, for each $\mathrm{P} \in\{\mathrm{A}, \mathrm{E}\}$. Finally, we write $\mathcal{G}_{1} \equiv \mathcal{G}_{2}$ when $\mathcal{G}_{1}, \mathcal{G}_{2}$ are relational basic power equivalent.

Relational basic power equivalence can be lifted to an equivalence relation between dynamic games in the same component-wise manner as before.

Our first observation is that the new notion solves our problem.

Fact 7.18 Relational basic power equivalence over $\mathbb{D}(\{\mathrm{A}, \mathrm{E}\}, O)$ is a congruence $\equiv$ with respect to the operations $\cup, \cap$, - plus sequential game composition $\circ$.

This motivates the following definition:

Definition 7.19 The dynamic game algebra $\Re$ of relational basic power equivalence (with outcome set $O$ ) is the quotient

$$
\langle\mathbb{D}(\{\mathrm{A}, \mathrm{E}\}, O), \cup, \cap,-, \circ\rangle / \equiv .
$$

But of course, this change in our set-up for defining basic powers needs to be checked for its compatibility with what went on before. We briefly state a number of results, whose proofs involve straightforward verifications:

Theorem 7.20 The following conditions capture representability of families of sets as relational basic powers in a game of imperfect information: Non-Emptiness, Consistency, Exhaustiveness, and one new property:

Union Closure: If all members of a family $F$ are relational basic powers of a player, then so is the union $\bigcup F$. 
Proof With Union Closure for two families of sets $\mathcal{X}$ and $\mathcal{Y}$, the basic powers of the two-player game constructed in the proof of Theorem 4.2 are closed under unions, which clearly means the basic powers of each player in that game coincide with their relational basic powers. Since the basic powers of the two players are the members of $\mathcal{X}$ and $\mathcal{Y}$ respectively, by Theorem 4.2 , the result follows.

We can also easily adapt our complete axiomatization of the modal logic of basic powers, so that it fits relational basic powers:

Theorem 7.21 The basic game logic BPL is also complete for basic relational powers in games, provided we add the axiom:

$$
\langle g, \mathrm{P}\rangle\left(\alpha ; \varphi_{1}\right) \wedge\langle g, \mathrm{P}\rangle\left(\beta ; \varphi_{2}\right) \rightarrow\langle g, \mathrm{P}\rangle\left(\alpha, \beta ; \varphi_{1} \vee \varphi_{2}\right)
$$

That is, the logic obtained by adding this axiom is sound and complete for validity over neighborhood models satisfying the extra constraint of Union Closure.

Proof The construction of a game in the proof of Theorem 6.1 stays exactly as before, but one has to check that the new axiom enforces Union Closure.

The basic game algebra changes in this new setting. We retain all valid equations from before, since we are dealing with a looser game equivalence when working with relational, rather than functional strategies. But the algebra remains weaker than the algebra of standard power equivalence.

\section{Fact 7.22}

1. All validities mentioned above for functional strategies are also valid with basic powers computed with relational strategies.

2. Propositional distribution is still not valid, but idempotence is.

Next, we turn to valid principles for composition of games, the motivation for making the change to relational strategies in the first place.

\section{Fact 7.23}

1. Game composition validates associativity, and left-distribution over choice:

$$
(x \cup y) \circ z=(x \circ z) \cup(y \circ z)
$$

2. Dual distributes over composition:

$$
-(x \circ y)=-x \circ-y
$$

3. Game composition does not validate right-distribution over choice.

Proof The first two items are immediate. Failure of right-distribution follows by the known fact that right-distribution fails already in the game algebra of standard power equivalence $[13,26]$. Since standard power equivalence is a coarser equivalence notion than relational basic power equivalence, any equation that fails in the game algebra of standard power equivalence also fails now. 
Open problem 7.24 Axiomatize the complete game algebra of the operations $\{\cup, \cap,-, \circ\}$ based on relational powers.

\section{Dynamic Game Logic and a New Game Algebra}

Various extensions are possible for the algebraic setting we have found so far. We mention two directions with further attractive tools for analyzing games.

\subsection{Dynamic Game Logic}

Putting instantial game logic together with game algebra yields a system in the style of dynamic game logic, [2, 19]. We now have instantial modalities describing basic powers of player $\mathrm{P}$ in games $\mathcal{G}$ that can have complex algebraic descriptions. This extension facilitates algebraic reasoning while offering more expressive power for what happens in the course of a game - advantages similar to those offered by PDL for the algebra of regular expressions:

$$
\langle\mathcal{G}, \mathrm{P}\rangle(\Psi ; \varphi)
$$

This formalism can be interpreted on our neighborhood models, provided these satisfy the constraint of Union Closure as well as the Non-Emptiness, Consistency and Exhaustiveness constraints. It is easy to lift the earlier definitions for the operations $\cup, \cap,-$ of initial choice and switch to definitions for power relations, [2, 19]. The further crucial point here is that, with the earlier obstacle to compositionally overcome, we can define the power relation for a composite game of the form $\mathcal{G}_{1} \circ \mathcal{G}_{2}$ in the following inductive manner:

$(u, Z) \in R_{\mathcal{G}_{1} ; \mathcal{G}_{2}}^{\mathrm{P}}$ iff $Z=\bigcup F$, for some family $F \subseteq \mathcal{P} W$ and some $Y \subseteq W$ with $(u, Y) \in R_{\mathcal{G}_{1}}^{\mathrm{P}}$ and $(Y, F) \in \widetilde{R}_{\mathcal{G}_{2}}^{\mathrm{P}}$,

where $(Y, F) \in \widetilde{R}_{\mathcal{G}_{2}}^{\mathrm{P}}$ means that the following back-and-forth conditions hold:

for all $y \in Y$ there is some $S \in F$ with $(y, S) \in R_{\mathcal{G}^{2}}^{\mathrm{P}}$ - and vice versa,

for all $S \in F$ with $(y, S) \in R_{\mathcal{G}^{2}}^{\mathrm{P}}$ there is some $y \in Y$ with $(y, S) \in R_{\mathcal{G}^{2}}^{\mathrm{P}}$.

This semantics validates principles that allow for recursive reasoning about basic game operations in a dynamic logic format:

\section{Reduction Axioms}

- $\langle-\pi, \mathrm{P}\rangle(\Psi ; \varphi) \leftrightarrow\langle\pi, \overline{\mathrm{P}}\rangle(\Psi ; \varphi)$

- $\left\langle\pi_{1} \cup \pi_{2}, \mathrm{P}\right\rangle(\Psi ; \varphi) \leftrightarrow\left\langle\pi_{1}, \mathrm{P}\right\rangle(\Psi ; \varphi) \vee\left\langle\pi_{2}, \mathrm{P}\right\rangle(\Psi ; \varphi)$

- $\left\langle\pi_{1} \cap \pi_{2}, \mathrm{P}\right\rangle(\Psi ; \varphi) \leftrightarrow \bigvee\left\{\left\langle\pi_{1}, \mathrm{P}\right\rangle\left(\Theta_{1} ; \varphi\right) \wedge\left\langle\pi_{2}, \mathrm{P}\right\rangle\left(\Theta_{2} ; \varphi\right) \mid \Psi=\Theta_{1} \cup \Theta_{2}\right\}$

- $\left\langle\pi_{1} \circ \pi_{2}, \mathrm{P}\right\rangle\left(\psi_{1}, \ldots, \psi_{n} ; \varphi\right) \leftrightarrow$

$$
\left\langle\pi_{1}, \mathrm{P}\right\rangle\left(\left\langle\pi_{2}, \mathrm{P}\right\rangle\left(\psi_{1} ; \varphi\right), \ldots,\left\langle\pi_{2}, \mathrm{P}\right\rangle\left(\psi_{n} ; \varphi\right) ;\left\langle\pi_{2}, \mathrm{P}\right\rangle \varphi\right)
$$

We call the extended logic DPL for "dynamic power logic". When added to our base logic BPL, these axioms lead to the following result. 
Theorem 8.1 The dynamic logic DPL of the operations $\{\cup, \cap,-, \circ\}$ with relational basic powers is completely axiomatizable.

Proof The crucial steps for a proof of this result are found in Appendix C.

From another angle, [3] explores the present perspective in more depth, in the form of a PDL-like dynamic logic over instantial neighborhood models with a new computational interpretation. This logic includes the game operations defined here, except for unrestricted dual, but it adds further constructions of test, and especially, finite iteration, which calls for new axioms and techniques.

\subsection{New Game Constructions and Extended Game Algebra}

The move to relational strategies solved a design problem, and led to an interesting game algebra and game logic. Even so, it is not the only possible approach. In particular, as noticed several times already, we have not deeply analyzed the role of imperfect information, and what repertoire of algebraic operations would best fit with that. Here is an example showing how a greater focus on imperfect information provides an alternative analysis of our earlier problems.

Example 8.2 (Restoring distribution through imperfect information) Consider our initial example of failure of the propositional distribution law with basic powers in Section 2. We insert one uncertainty link in the game to the right, as depicted in Fig. 9. Now, keeping in mind that strategies in imperfect information games must choose the same moves at epistemically linked points, it is easy to see that basic powers are the same in the two games shown:

This simple observation suggests a natural new choice operation that combines imperfect information games, namely, one that introduces uncertainty:

Let $\mathcal{G}_{1} \cup \mathcal{G}_{2}$ be the game where A starts by choosing either $\mathcal{G}_{1}$ or $\mathcal{G}_{2}$, but this choice is not observed by $A$ - and likewise for $\mathcal{G}_{1} \cap \mathcal{G}_{2}$.

The game algebra with 'partly invisible choices' that now results has operations

$$
\{-, \cup, \underline{\cup}, \cap, \underline{\cap}, \circ\}
$$

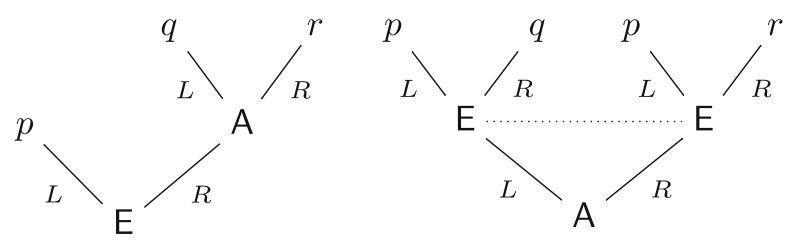

Fig. 9 Distribution revisited 
Interestingly, it now has valid equations in the extended vocabulary that take the place of the invalid distribution law, such as

$$
x \cap(y \cup z)=(x \cap y) \underline{\cup}(x \cap z) .
$$

This new game algebra provides a much tighter fit for our earlier game transformations. Its extended propositional logic has strong analogies with the 'Thompson transformations' for imperfect information games in game theory, cf. [1]. But we do not have a full-fledged theory to offer at this stage.

Open problem 8.3 Axiomatize the complete algebra of $\{-, \cup, \underline{\cup}, \cap, \underline{\cap}, \circ\}$.

This game algebra can be viewed as a generalized propositional logic. It has even intriguing stronger principles than the one we noted. Here is one very strong distributivity principle without a classical propositional counterpart.

Fact 8.4 The following equivalence is valid: $(x \cap y) \underline{\cup}(z \cap u)=(x \cup z) \cap(y \cup u)$.

Elaborating the syntax of this propositional logic takes a good deal of care, since in stating valid principles, we need to 'balance' formulas, and mark subformulas (viewed as possible moves) in a way that respects the uniformity conditions on strategies in imperfect information games (indistinguishable positions should have the same moves available, strategies should make the same choice at indistinguishable positions). We will not pursue this system here, but note that there are analogies with 'IF-logic' of imperfect information, as pointed out in van Benthem [2, Chapter 21]. The latter source also suggests adding even one more operation to the algebra, namely parallel composition of games.

As before, we should also add sequential composition to the signature of this algebra. In that case, another suggestive observation can be made. Imperfect information can play a similar role to our introduction of relational strategies.

Example 8.5 (Safety revisited) Consider the example of failure of safety given in the proof of Fact 7.16, which blocked compositionality for basic powers with functional strategies. This time, however, assume that in the duplicated model, there is an uncertainty link between the two copies of the midlevel point (Fig. 10). Now the only admissible strategies are those that make the same choice at both midlevel points, and accordingly, the resulting basic powers are the same in both models.

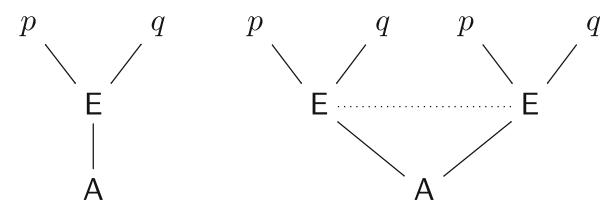

Fig. 10 Safety revisited 
We have not been able to determine precisely to which extent imperfect information, made explicit in the manner proposed above, can play the same role in the design of game algebra and dynamic game logic as our move from functional to relational strategies. Both approaches have their own motivation, but we leave a deeper comparison of the two as an open problem.

Finally, we started this section by saying that imperfect information games are a typical challenge to compositional algebraic thinking because of the arbitrary crossing of uncertainty lines between subtrees, making the notion of a subgame problematic. The way we got around this was by having algebraic operations that only introduce epistemic uncertainty in very controlled ways. We will discuss a few further aspects of this setting in Section 9.1 below.

\section{Further Directions}

In this final section, we collect a few further perspectives on our motivating issue of natural game equivalences that form a background to what we have done in this paper. Our discussion will be light, and we mainly raise new questions.

\subsection{Equivalence Levels and Translating Logics}

We have seen various natural levels for studying games, defined by invariance notions, and we gave matching logics. The resulting total picture raises questions. How are the various logics that we considered connected? Can we switch between levels in a systematic manner, perhaps via translations of logics?

We look at these issues for finite game trees with perfect information. Imperfect information will be discussed in Section 9.2. Here is a result from [2].

Fact 9.1 The neighborhood modality $\langle g, \mathrm{P}\rangle \varphi$ for standard powers is definable in the modal (fixpoint) language of actions by the following equivalence, where standard modalities $\diamond, \square$ run over all available moves for the players $\mathrm{A}$ and $\mathrm{E}$ :

$$
\langle g, \mathrm{P}\rangle \varphi \leftrightarrow v x .\left(\varphi \vee\left(\text { turn }_{\mathrm{P}} \wedge \diamond x\right) \vee\left(\operatorname{turn}_{\overline{\mathrm{P}}} \wedge \square x\right)\right)
$$

This logical pattern of alternating existential and universal modalities over moves is characteristic of many game-theoretic arguments. It follows that standard powers are invariant for standard modal bisimulations of finite game-trees.

However, with basic powers, this changes: these are not invariant for bisimulation. In fact, interpreting the example shown in Fig. 8 of Section 7.2 in a different way, it shows precisely that. The two games are bisimilar as trees, but do not have the same basic powers. Stated in terms of our language,

Fact 9.2 The BPL-formula $\langle g, \mathrm{E}\rangle(p, q ; p \vee q)$ is not invariant for standard bisimulation, and hence it is not definable in the modal base language of games.

One symptom of this is the impossibility of generalizing the earlier recursion for neighborhood modalities to the INL-modality with instantial information. More 
generally, the second-order quantification over strategies as functions goes beyond the basic modal language.

However, things change with basic powers based on relational strategies.

Fact 9.3 Relational basic powers are invariant for standard bisimulation.

Proof It is easy to see that any two bisimilar game trees can be turned into isomorphic trees by simply creating sufficiently many copies of the moves of each player. This construction obviously does not change the relational basic powers of a game (though it does change the basic powers), and the result follows.

Accordingly, we can provide an explicit definition of the instantial neighborhood modality for relational basic powers in the modal $\mu$-calculus. For the sake of simplicity, we focus on the case with only a single existential witness formula, and the relevant equivalence is then as follows:

$$
\left.\langle g, \mathrm{P}\rangle(\psi ; \varphi) \leftrightarrow v x .(\psi \wedge \varphi) \vee\left(\operatorname{turn}_{\mathrm{P}} \wedge \diamond x\right) \vee\left(\operatorname{turn}_{\overline{\mathrm{P}}} \wedge \square\langle g, \mathrm{P}\rangle \varphi \wedge \diamond x\right)\right)
$$

where we use the definition of the formula $\langle g, \mathrm{P}\rangle \varphi$ from Fact 9.1. The equivalence stated here follows by an easy inspection of the three relevant cases in game trees. The reduction is slightly more complex to state with more witness formulas $\psi-$ as would be needed, e.g., to analyse how the earlier non-bisimulation invariant formula $\langle g, \mathrm{E}\rangle(p, q ; p \vee q)$ becomes definable in this new setting. ${ }^{4}$

\section{Coda: Logics for Strategic Forms}

Finally, we mention one more level of looking at games, the strategic forms that were linked to basic power analysis in Section 4.2. These matrix games look like simple games of imperfect information, and this is a valid analogy. Indeed, they have a simple bimodal logic for one-step simultaneous action where one modality ranges over choices of a player $i$ ( $i$ 's 'freedom') and the other over choices of the other player (that is, $i$ 's uncertainty), cf. [2]. How is this new logic related to our earlier ones? This issue is more delicate. Points in the strategic form are strategy profiles, higherorder objects from the perspective of extensive game trees that cannot be identified with nodes or subtreees. Therefore, we do not expect simple translations between the logics of extensive games and strategic form games, and we leave a complete analysis for future study.

\subsection{Imperfect Information and Epistemic Logic}

From a logical point of view, imperfect information games combine two relations: actions and uncertainty links. This suggests extending our earlier languages to a

\footnotetext{
${ }^{4}$ Our fixed-point formula also suggests the possible interest of a combined logic of standard monotonic powers and basic powers.
} 
richer bimodal logic that can talk about action, or at a coarser level: powers, via suitable modalities, say in INL, but that also has an epistemic operator $K \varphi$ for knowledge. This interplay is important because we can then formulate non-trivial statements about the interplay of knowledge and action, such as

- $K_{\mathrm{A}}\langle g, \mathrm{E}\rangle \varphi$ : player A knows $\mathrm{E}$ has a power for achieving the truth of $\varphi$,

- $\quad\langle g, \mathrm{~A}\rangle K_{\mathrm{E}} \varphi$ : player A has a power for making player $\mathrm{E}$ know that $\varphi$.

Example 9.4 Natural axioms formulated in such a bimodal language express special properties one can assume about players, such as (in simplified form)

$$
K_{\mathrm{P}}\langle g, \mathrm{P}\rangle \varphi \rightarrow\langle g, \mathrm{P}\rangle K_{\mathrm{P}} \varphi
$$

This axiom says essentially that actions that can be perfectly observed do not increase uncertainty, a version of Perfect Recall for players, cf. [2] for details. The converse implication also has a clear game-theoretic meaning:

$$
\langle g, \mathrm{P}\rangle K_{\mathrm{P}} \varphi \rightarrow K_{\mathrm{P}}\langle g, \mathrm{P}\rangle \varphi
$$

This expresses essentially that learning cannot take place spontaneously without a difference in action, a property of players sometimes called No Miracles.

Combined logics of action and knowledge are well-understood in terms of expressive power and of computational complexity. In particular, [1] ties Perfect Recall and No Miracles to a style of play where uncertainty links in a game tree arise by an update mechanism from dynamic-epistemic logic. Both players can observe each other's moves wholly or partially, which creates horizontal uncertainty links only, resulting in a synchronous view of the game. This is precisely the view of games encoded in our earlier game algebra of $\{-, \cup, \underline{\cup}, \cap, \underline{\cap}, \circ\}$.

We will not pursue this epistemic logic, but it relates to many earlier topics. For instance, strategies in games of imperfect information are like 'knowledge programs', where players' choices are guided only by what they know. Also, issues of definability between levels of representing games get more complicated.

But perhaps the major problem left open here concerns our initial topic of game equivalence. Suppose we are presented with two extensive games annotated with uncertainty links indicating the information available to players at various stages of the game. When do we consider these games to be the same? This is not just a matter of comparing actions or powers in some absolute sense. It also depends on something beyond the explicit game structure, namely, what we assume about the type of players inhabiting these games. For instance, two games may be the same for rational players following Backward Induction reasoning, but not for players following some other method of game solution, say, bounded automata restricted to using very simple strategies. Perhaps we need to refine our earlier intuitions then to study equivalence of games "as played", taking the style of analysis in this paper to a richer agent-oriented setting. 


\subsection{Preference and rationality}

As a final topic, we mention one more crucial feature of actual games: players' preferences. Behavior in games, and social behavior generally, involves a balance between available actions, knowledge and belief with players' preferences between outcomes. A standard game-theoretic solution method showing this mixture of these crucial agent features at work is the following procedure:

Example 9.5 (Backward Induction) To show the preferences in the games depicted in Fig. 11, outcomes have been marked numerically at the three endpoints $y, u, v$ in the order (value for A, value for E).

Here, a rational player $E$ will go left at his turn, since he prefers the outcome 3 over the 2 to the right. Then player A, knowing (or believing) this, will go left as well, as she prefers outcome 1 to 0 . Thus, the Backward Induction strategy for A is "left", and so is that for E - though the latter strategy never gets played.

Backward Induction has long been a benchmark for logical studies of games, [2], and there is an extensive theory of this and other solution methods. In particular, the reasoning leading to the above prediction about the game has been analyzed in fixedpoint logics for relational, rather than functional strategies, a move similar to that in Section 9.2. The Backward Induction reasoning can also be captured in a bimodal logic of actions with an added preference modality.

We conclude this preliminary discussion by connecting with our theme of powers. The above computation can also be seen as a process of "pruning powers", more precisely, of intermediate powers in the sense of Section 4.2.

Example 9.6 (Backward Induction and powers) At point $z$ in the game tree of Fig. 11, $\mathrm{E}$ has powers $\{u\}$ and $\{v\}$. But these powers stand in a preference relation $\{u\}>_{\mathrm{E}}\{v\}$ in an obvious sense. We can lift players' preference relation to any two sets $X, Y$ as follows:

$$
X>_{\mathrm{p}} Y
$$

if all points in $X$ are preferred to all points in $Y$. There are other ways of lifting relations on points to relations on sets, but this one suffices for our purposes.

The rationality embodied in the Backward Induction algorithm assumes essentially that "players never play a power for which they have a better one" in this lifted sense. Continuing in this way along the above reasoning steps, a subtle change takes place. Player A now needs to compare, not $\{y\}$ and $\{u, v\}$, but $\{y\}$ and $\{u\}$, where $\{u\}$

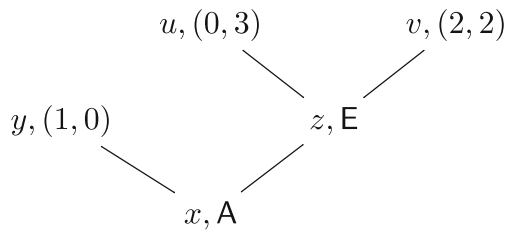

Fig. 11 Backward induction 

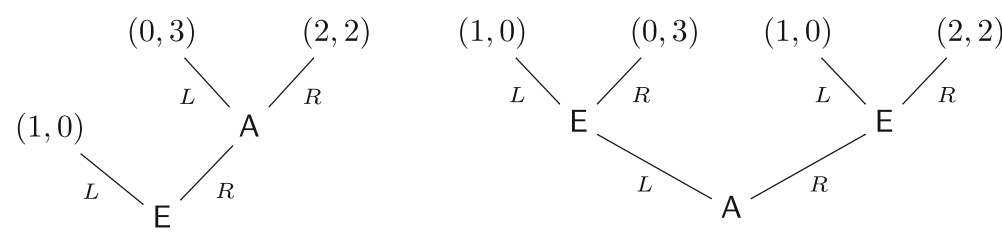

Fig. 12 Distribution with preference

is the prediction or a belief about best play further on. But then, since $\{x\}>_{\mathrm{A}}\{u\}$, her best power is $\{x\}$.

Thus we must extend our earlier analysis of games in terms of players' basic powers to include their best or optimal powers. We do not yet know how to do this in a satisfying manner, so we leave it as an open problem.

We end with an observation connecting to our earlier concerns.

Example 9.7 (Failure of distribution with preference) Applying our running example of the propositional distribution law to the preceding game, yields the new extensive game depicted in Fig. 12.

Now compare the Backward Induction outcomes for these two games. In the game to the right, the Backward Induction outcome is $(2,2)$, and clearly, this is not the same prediction as for the game on the left, which is $(1,0)$.

So, distribution fails for games with preference, but for other reasons than we had before. The game algebra of games under preference seems a good place for studying the effects of considering players' preferences explicitly. ${ }^{5}$

In all, adding preferences poses a further challenge to our style of analysis. The issue is not a scarcity of formal preference logics, but one of clarity on the entanglement of action, belief and preference that drives social behavior. And at the more technical level of game equivalence, an earlier problem becomes even more acute. When we call two games equivalent, what type of players do we have in mind, satisfying what sort of entanglement assumptions?

\section{Conclusion}

We have defined a new game equivalence in terms of basic powers, and developed its modal logic and game algebra. In the process, we found analogues of results for other game equivalences, but also a number of non-trivial new features that suggest new lines of investigation on logic and games, such as the game algebra of operations that take on board imperfect information in various ways.

While we consider basic power equivalence well-motivated, there can be yet further natural game equivalences. As we have noted, the search for mathematical

\footnotetext{
${ }^{5}$ Another source of inspiration for further logical analysis here may be the work on so-called 'Nashconsistent powers' in game theory, [22], and in coalition logic, [16].
} 
structure levels in a rich domain is in principle open-ended. In that sense, this article is a pilot study for more generally useful logical techniques. In addition, we have also briefly noted, though not explored in depth, a second role for logic, now more as a guardian of coherence: namely, studying translations and forms of tracking between different representation levels for games.

Even so, our style of analysis has clear limitations. We considered two-player games only, and we mainly looked at finite instead of infinite games where the histories themselves are the output of the game. And we encountered a serious barrier to power analysis when discussing games with preferences. We do not see these limitations as definitive, but clearly, much needs to be done before we can assess the true merits of the logical perspective pursued in this paper.

Acknowledgments We thank audiences in Amsterdam and at TARK 2017 Liverpool for their feedback, in particular, Valentin Goranko and Paolo Turrini. We also thank the two referees of this paper for their careful and helpful comments.

Open Access This article is distributed under the terms of the Creative Commons Attribution 4.0 International License (http://creativecommons.org/licenses/by/4.0/), which permits unrestricted use, distribution, and reproduction in any medium, provided you give appropriate credit to the original author(s) and the source, provide a link to the Creative Commons license, and indicate if changes were made.

\section{Appendix A: Selected Game-Theoretical Definitions}

Definition A.1 Let a tree $\mathcal{T}$ over a given set $\Sigma$ be a non-empty and prefix closed subset of the set $\Sigma^{*}$ of finite words over the alphabet $\Sigma$. In particular the empty word $\varepsilon$ belongs to every tree and is called the root of the tree.

Standard concepts like branches and leaves of trees are defined as usual.

Definition A.2 An extensive game $\mathcal{G}$ for a finite set of players $A$ with outcomes in the set $O$ is a tuple $(\mathcal{T}, t, o, \Pi)$ where $\mathcal{T}$ is a tree over some set $\Sigma, t$ a map from non-leaf nodes of $\mathcal{T}$ to $A, o$ a map from branches of $\mathcal{T}$ to $O$, and $\Pi$ a partition of $\mathcal{T}$ subject to the following condition: for any pair $w, v$ within the same partition cell of $\Pi$, we have $t(n)=t\left(n^{\prime}\right)$ and for all $i \in \Sigma$ :

$$
w \cdot i \in \mathcal{T} \text { iff } v \cdot i \in \mathcal{T} \text {. }
$$

If all partition cells of $\Pi$ are singletons we call $\mathcal{G}$ a game of perfect information, and we omit $\Pi$.

Maximal branches of $\mathcal{T}$ will also be called full matches, and prefixes of maximal branches are called partial matches.

A strategy for player $a \in A$ is a map $\sigma: t^{-1}[a] \rightarrow \Sigma$ where $w \cdot \sigma(w)$ is a child of $w$ for each $w$ with $t(w)=a$, and $\sigma(w)=\sigma\left(w^{\prime}\right)$ whenever $w, w^{\prime}$ are in the same partition cell in $\Pi$. A strategy profile is a tuple $\left(\sigma_{a}\right)_{a \in A}$ of one strategy for each player in $A$. A strategy profile $p$ determines a unique full match guided by the strategies of each player, so we can speak of the outcome of the profile $p$ and denote it by $o(p)$. Generally, we say that a full match $m$ of $\mathcal{G}$ is guided by the strategy $\sigma$ for $a$ if for 
every prefix $w$ of $m$ such that $t(w)=a, \sigma(w)$ is also a prefix of $m$. Match $(\sigma)$ is the set of $\sigma$-guided matches.

$\mathbb{G}(A, O)$ is the set of games for players $A$ with outcomes in $O$. For two-player games we call the players $E$ and $A$. We set $\bar{E}=A$ and $\bar{A}=E$.

We have not attributed payoffs to matches in a game or preferences over the outcomes, but rather (and more generally) outcomes from some fixed set. In this sense we are dealing with game forms rather than proper games.

Definition A.3 Let $\mathcal{G}=(\mathcal{T}, t, o, \Pi)$ be a game with outcomes in $O$. A set $P \subseteq O$ is a power of player $a \in A$ in the game $\mathcal{G}$ if there is a strategy $\sigma$ for $a$ in $G$ such that $o(m) \in P$ for every $\sigma$-guided match $m$. Given a player $a \in A$ we let $P_{a}(\mathcal{G})$ denote the set of powers of $a$ in $\mathcal{G}$.

Two games $\mathcal{G}_{1}, \mathcal{G}_{2} \in \mathbb{G}(A, O)$ are power equivalent, $\mathcal{G}_{1} \sim \mathcal{G}_{2}$, if for all $a \in A$ : $P_{a}\left(\mathcal{G}_{1}\right)=P_{a}\left(\mathcal{G}_{2}\right)$. If $P_{a}\left(\mathcal{G}_{1}\right)=P_{a}\left(\mathcal{G}_{2}\right)$ for some specific $a \in A$, we write $\mathcal{G}_{1} \sim_{a} \mathcal{G}_{2}$.

\section{Appendix B: Soundness of Principles for Basic Powers}

Proof of Fact 4.1 For Non-Emptiness, pick a player P. By definition of an extensive game, the set of strategies available to each player is non-empty: in particular, if $t^{-1}[\mathrm{P}]=\emptyset$ then the empty function is the unique available strategy for $\mathrm{P}$. So pick an arbitrary strategy $\sigma$ for $\mathrm{P}$. Then the set $\{o(m) \mid m \in \operatorname{Match}(\sigma)\}$ is a basic power for $\mathrm{P}$.

For Consistency, let $P$ be a basic power for $\mathrm{E}$ and $Q$ a basic power for A. Then there are strategies $\sigma, \tau$ for E, A respectively such that $P=\{o(m) \mid m \in \operatorname{Match}(\sigma)\}$ and $Q=\{o(m) \mid m \in \operatorname{Match}(\tau)\}$. Hence $o(p) \in P \cap Q$ for the strategy profile $p=(\sigma, \tau)$.

Finally, for Exhaustiveness, suppose that $P$ is a basic power of Player $\mathrm{P}$ and that $x \in P$. Pick a strategy $\sigma$ for $\mathrm{P}$ such that $P=\{o(m) \mid m \in \operatorname{Match}(\sigma)\}$. Since $x \in P$, there is a $\sigma$-guided match $m$ such that $o(m)=x$. We define a strategy $\tau$ for Player $\overline{\mathrm{P}}$ as follows: if a position $u \in t^{-1}[\overline{\mathrm{P}}]$ belongs to $m$ then $u$ cannot be an endpoint of he game, since $t(u)$ is defined, and hence, because $m$ is a full match, there must be some unique child $v$ of $u$ such that $v$ also belongs to $m$. Set $\tau(u)=v$. If $u \in t^{-1}[\overline{\mathrm{P}}]$ does not belong to $m$ then define $\tau(u)$ arbitrarily. Then $m$ is a $\tau$-guided match, so $x=o(m) \in Q$ where $Q=\{o(m) \mid m \in \operatorname{Match}(\tau)\}$, which is a basic power of $\overline{\mathrm{P}}$.

\section{Appendix C: Completeness of Dynamic Game Logic}

We denote the axiom system in Section 8.1 by Ax2 and write Ax2 $\vdash \varphi$ to say that formula $\varphi$ is provable in this axiom system. We also write $\varphi \vdash_{\text {Ax2 }} \psi$ for Ax2 $\vdash \varphi \rightarrow$ $\psi$. We sometimes drop the reference to Ax2 to keep notation cleaner.

Proposition C.1 (Soundness) If Ax $\vdash \varphi$, then $\varphi$ is valid on all neighborhood models for games with forcing relations as defined earlier. 
Proof We consider only soundness of the reduction axioms. Soundness of the axiom for dual - is immediate from the forcing definition for game dual reversing the roles of the players: in fact, the axiom encodes that definition. The same is true for the initial choice operation $\llbracket \cup \rrbracket$ whose axiom is immediate from the game construction. For the dual construction $\llbracket \cap \rrbracket$, we reason as follows.

Suppose that $\mathfrak{M}, w \Vdash\left\langle\pi_{1} \cap \pi_{2}\right\rangle(\Psi ; \varphi)$. Then there is some set $Z$ such that $(w, Z) \in R_{\pi_{1} \cap \pi_{2}}, Z \subseteq \llbracket \varphi \rrbracket$ and $Z \cap \llbracket \psi \rrbracket \neq \emptyset$ for all $\psi \in \Psi$. Hence $Z$ is of the form $Z_{1} \cup Z_{2}$ where $\left(w, Z_{1}\right) \in R_{\pi_{1}}$ and $\left(w, Z_{2}\right) \in R_{\pi_{2}}$. Let $\Theta_{1}=\left\{\psi \in \Psi \mid Z_{1} \cap \llbracket \psi \rrbracket \neq\right.$ $\emptyset\}$, and let $\Theta_{2}=\left\{\psi \in \Psi \mid Z_{2} \cap \llbracket \psi \rrbracket \neq \emptyset\right\}$. Then, since $Z=Z_{1} \cup Z_{1}$, we have $\Psi=\Theta_{1} \cup \Theta_{2}$. Furthermore, we get

$$
\mathfrak{M}, w \Vdash\left\langle\pi_{1}\right\rangle\left(\Theta_{1} ; \varphi\right) \wedge\left\langle\pi_{2}\right\rangle\left(\Theta_{2} ; \varphi\right)
$$

as required. The converse direction is proved in a similar manner.

Next, we consider sequential composition. For one direction of the equivalence, suppose that $\mathfrak{M}, w \Vdash\left\langle\pi_{1} ; \pi_{2}\right\rangle\left(\psi_{1}, \ldots, \psi_{n} ; \varphi\right)$. Then there is some set $Z$ with $(w, Z) \in R_{\left\langle\pi_{1} ; \pi_{2}\right\rangle}, Z \subseteq \llbracket \varphi \rrbracket$ and $Z \cap \llbracket \psi_{i} \rrbracket \neq \emptyset$ for each $\psi_{i}$. By definition of the composition operator, we find a set $Y$ with $(w, Y) \in R_{\pi_{1}}$ and a family of sets $F$ such that $(Y, F) \in \bar{R}_{\pi_{2}}$ and $Z=\bigcup F$. So for each $v \in Y$ there is some $S_{v} \in F$ with $\left(v, S_{v}\right) \in R_{\pi_{2}}$, and we get $S_{v} \subseteq \llbracket \varphi \rrbracket$ so $\mathfrak{M}, v \Vdash\left\langle\pi_{2}\right\rangle \varphi$. Also, for each $\psi_{i}$ there is some $S_{i} \in F$ with $S_{i} \cap \llbracket \psi_{i} \rrbracket \neq \emptyset$, and there must be some $v \in Y$ with $\left(v, S_{i}\right) \in R_{\pi_{2}}$, hence $\mathfrak{M}, v \Vdash\left\langle\pi_{2}\right\rangle\left(\psi_{i} ; \varphi\right)$. It follows that $\mathfrak{M}, w \Vdash\left\langle\pi_{1}\right\rangle\left(\left\langle\pi_{2}\right\rangle\left(\psi_{1} ; \varphi\right), \ldots,\left\langle\pi_{2}\right\rangle\left(\psi_{n} ; \varphi\right) ;\left\langle\pi_{2}\right\rangle \varphi\right)$ as required.

Conversely, suppose that $\mathfrak{M}, w \Vdash\left\langle\pi_{1}\right\rangle\left(\left\langle\pi_{2}\right\rangle\left(\psi_{1} ; \varphi\right), \ldots,\left\langle\pi_{2}\right\rangle\left(\psi_{n} ; \varphi\right) ;\left\langle\pi_{2}\right\rangle \varphi\right)$. Then there exists some set $Y$ such that $(w, Y) \in R_{\pi_{1}}, Y \subseteq \mathbb{U}\left\langle\pi_{2}\right\rangle \varphi \rrbracket$ and $Y \cap$ $\llbracket\left\langle\pi_{2}\right\rangle\left(\psi_{i} ; \varphi\right) \rrbracket \neq \emptyset$ for each $i \in\{1, \ldots, n\}$. Let:

$$
F:=\left\{Z \subseteq W \mid Z \subseteq \llbracket \varphi \rrbracket \&(v, Z) \in R_{\pi_{2}} \text { for some } v \in Y\right\}
$$

Since $Y \subseteq \mathbb{I}\left\langle\pi_{2}\right\rangle \varphi \rrbracket$, we get $(Y, F) \in \widetilde{R}_{\pi_{2}}$, so $(w, \bigcup F) \in R_{\pi_{1} ; \pi_{2}}$. Furthermore, since $Y \cap \llbracket\left\langle\pi_{2}\right\rangle\left(\psi_{i} ; \varphi\right) \rrbracket \neq \emptyset$ for each $i \in\{1, \ldots, n\}$, it follows that $\bigcup F \cap \llbracket \psi_{i} \rrbracket \neq \varnothing$ for each $i \in\{1, \ldots, n\}$. Hence $\mathfrak{M}, w \Vdash\left\langle\pi_{1} ; \pi_{2}\right\rangle\left(\psi_{1}, \ldots, \psi_{n} ; \varphi\right)$ as required.

Next, by applying the sound reduction axioms, a standard argument shows that, for every consistent formula $\varphi$ of DPL there is a provably equivalent equivalent formula $\varphi^{t}$ in INL, which is then satisfiable by Theorem 6.1.

The preceding argument establishes the following result.

Theorem C.2 (Completeness) A formula $\varphi$ of DPL is valid on all neighborhood models iff $\mathrm{Ax} 2 \vdash \varphi$.

Also, the finite model property and decidability carry over from INL:

Theorem C.3 The logic DPL has the finite model property and is decidable.

This simple completeness argument no longer works with iteration, cf. [3]. 


\section{References}

1. van Benthem, J. (2001). Games in dynamic-epistemic logic. Bulletin of Economic Research, 53(4), 219-248.

2. van Benthem, J. (2014). Logic in games. Cambridge: MIT Press.

3. van Benthem, J., Bezhanishvili, N., Enqvist, S. (2017). A propositional dynamic logic for instantial neighbourhood semantics. ILLC Preprint PP-2018-1. To appear in Studia Logica.

4. van Benthem, J., Bezhanishvili, N., Enqvist, S., Yu, J. (2017). Instantial neighborhood logic. The Review of Symbolic Logic, 10(1), 116-144.

5. Bergstra, J.A., Ponse, A., Smolka, S.A. (2001). Handbook of process algebra. Amsterdam: Elsevier.

6. Bezhanishvili, N. (2006). Lattices of intermediate and cylindric modal logics. Dissertation 2006-02. Institute for Logic, Language and Computation, University of Amsterdam.

7. Blackburn, P., de Rijke, M., Venema, Y. (2001). Modal logic. Cambridge: Cambridge University Press.

8. Bonanno, G. (1992). Set-theoretic equivalence of extensive-form games. International Journal of Game Theory, 20(4), 429-447.

9. Burris, R., \& Sankappanavar, H. (1981). A course in universal algebra. Heidelberg: Springer.

10. Elmes, S., \& Reny, P.J. (1994). On the strategic equivalence of extensive form games. Journal of Economic Theory, 62(1), 1-23.

11. Gabbay, D.M., Kurucz, A., Wolter, F., Zakharyaschev, M. (2003). Many-dimensional modal logics. Studies in logic and the foundations of mathematics Vol. 148. Amsterdam: North-Holland Publishing Co.

12. Gärdenfors, P. (1981). Rights, games and social choice. Noûs, 15, 341-356.

13. Goranko, V. (2003). The basic algebra of game equivalences. Studia Logica, 75(2), 221-238.

14. Goranko, V., Jamroga, W., Turrini, P. (2013). Strategic games and truly playable effectivity functions. Journal of Autonomous Agents and Multi-Agent Systems, 26, 288-314.

15. Grossi, D., \& Turrini, P. (2012). Short sight in extensive games. In van der Hoek, W., Padgham, L., Conitzer, V., Nikoff, M.W. (Eds.) Proceedings AAMAS XI (pp. 805-812). Valencia.

16. Hansen, H., \& Pauly, M. (2002). Axiomatising nash-consistent coalition logic. In Proceedings JELIA 2002 (pp. 394-406). Springer.

17. Mailath, G.J., Samuelson, L., Swinkels, J.M. (1994). Normal form structures in extensive form games. Journal of Economic Theory, 64(2), 325-371.

18. Osborne, M., \& Rubinstein, A. (1994). A course in game theory. Cambridge MA: The MIT Press.

19. Parikh, R. (1985). The logic of games and its applications. Annals of Discrete Mathematics, 24, 111139.

20. Pauly, M. (2001). Logic for social software. PhD thesis, ILLC University of Amsterdam.

21. Peleg, B. (1998). Effectivity functions, game forms, games, and rights. Social Choice and Welfare, 15, 67-80.

22. Peleg, B., \& Peters, H. (2010). Strategic social choice. Stable representations of constitutions, (p. 2424). Springer, Lecture Notes in Computer Science.

23. Thompson, F. (1997). Equivalence of games in extensive form. Classics in Game Theory, 36.

24. van der Hoek, W., \& Pauly, M. (2007). Modal logic for games and information. In Blackburn, P., van Benthem, J., Wolter, F. (Eds.) Handbook of modal logic, studies in logic and practical reasoning, (Vol. 3 pp. 1077-1148). Amsterdam: Elsevier Science Publishers.

25. Venema, Y. (1995). Cylindric modal logic. Journal of Symbolic Logic, 60(2), 591-623.

26. Venema, Y. (2003). Representation of game algebras. Studia Logica, 75(2), 239-256. 